\title{
A study on the fourth $q$-Painlevé equation
}

\author{
Kenji KAJIWARA ${ }^{1)}$, Masatoshi NOUMI ${ }^{2)}$ and Yasuhiko YAMADA ${ }^{2)}$ \\ 1) Department of Electrical Engineering, Doshisha University \\ Kyotanabe, Kyoto 610-0321, JAPAN \\ 2) Department of Mathematics, Kobe University \\ Rokko, Kobe 657-8501, JAPAN
}

August 23, 2019

\begin{abstract}
A $q$-difference analogue of the fourth Painlevé equation is proposed. Its symmetry structure and some particular solutions are investigated.
\end{abstract}

\section{Introduction}

The importance of six Painlevé equations and their solutions in mathematics and mathematical physics is widely accepted. The discrete analogue of Painlevé equations has been first recognized in [6, 7, 8] where they appear as Schlesinger transformations of the Painlevé equations. After the discovery of discrete analogue of the Painlevé property which is now called the singularity confinement property偖, a lot of second order difference equations, including $q$-difference equations, have been identified as the discrete Painlevé equations 16, 5. By investigating the Lax pairs, particular solutions including $\tau$ functions and bilinear equations, and so on [3], it has been gradually recognized that discrete Painlevé equations admit similar properties to original Painlevé equations.

The purpose of this paper is to introduce a $q$-difference analogue of the fourth Painleve equation $\mathrm{P}_{\mathrm{IV}}$. This equation, called the $q$-Painlevé IV equation $q \mathrm{P}_{\mathrm{IV}}$ below, shares many characteristic properties with the original $\mathrm{P}_{\mathrm{IV}}$. In particular, it admits the action of the affine Weyl group of type $A_{2}^{(1)}$ as a group of Bäcklund transformations. Furthermore, it has an analogue of classical solutions expressible by the continuous $q$-HermiteWeber functions and rational solutions corresponding those of $\mathrm{P}_{\mathrm{IV}}$ studied in [15, 10, 13].

The plan of this paper is as follows. We introduce our $q \mathrm{P}_{\mathrm{IV}}$ in Section 1 and describe its symmetry structure in terms of the affine Weyl group $W\left(A_{2}^{(1)}\right)$. In Section 2, we construct a $q$-analogue of classical solutions along each reflection hyperplane from a seed solution which is described by a discrete Riccati equation. Each particular solutions of this class is expressed in terms of a Toeplitz type determinant of continuous $q$-HermiteWeber functions. In Section 3, we discuss some related topics including the relationship between our $q \mathrm{P}_{\text {IV }}$ and Sakai's Mul.6 system [17] and the ultra-discrete limit of $q \mathrm{P}_{\mathrm{IV}}$.

\section{Symmetric form of a $q$-Painlevé equation $q \mathbf{P}_{\mathrm{IV}}$}




\section{$1.1 \quad q$-Painlevé equation $q \mathbf{P}_{\mathrm{IV}}$}

In this paper, we consider the following discrete system including three dependent variables $f_{0}, f_{1}, f_{2}$ and three parameters $a_{0}, a_{1}, a_{2}$ :

$$
\begin{aligned}
& \overline{a_{0}}=a_{0}, \quad \overline{a_{1}}=a_{1}, \quad \overline{a_{2}}=a_{2}, \\
& \overline{f_{0}}=a_{0} a_{1} f_{1} \frac{1+a_{2} f_{2}+a_{2} a_{0} f_{2} f_{0}}{1+a_{0} f_{0}+a_{0} a_{1} f_{0} f_{1}}, \\
& \overline{f_{1}}=a_{1} a_{2} f_{2} \frac{1+a_{0} f_{0}+a_{0} a_{1} f_{0} f_{1}}{1+a_{1} f_{1}+a_{1} a_{2} f_{1} f_{2}}, \\
& \overline{f_{2}}=a_{2} a_{0} f_{0} \frac{1+a_{1} f_{1}+a_{1} a_{2} f_{1} f_{2}}{1+a_{2} f_{2}+a_{2} a_{0} f_{2} f_{0}},
\end{aligned}
$$

where ${ }^{-}$stands for the discrete time evolution. We will also use the notation $t(x)=\bar{x}$ when we regard $x \rightarrow \bar{x}$ as a transformation of variables. The inverse transformation of $t$ is given by

$$
\begin{aligned}
& \underline{a_{0}}=a_{0}, \quad \underline{a_{1}}=a_{1}, \quad \underline{a_{2}}=a_{2}, \\
& \underline{f_{0}}=\frac{f_{2}}{a_{0} a_{1}} \frac{a_{0} a_{1}+a_{0} f_{1}+f_{0} f_{1}}{a_{2} a_{0}+a_{2} f_{0}+f_{2} f_{0}}, \\
& \underline{f_{1}}=\frac{f_{0}}{a_{1} a_{2}} \frac{a_{1} a_{2}+a_{1} f_{2}+f_{1} f_{2}}{a_{0} a_{1}+a_{0} f_{1}+f_{0} f_{1}}, \\
& \underline{f_{2}}=\frac{f_{1}}{a_{2} a_{0}} \frac{a_{2} a_{0}+a_{2} f_{0}+f_{2} f_{0}}{a_{1} a_{2}+a_{1} f_{2}+f_{1} f_{2}} .
\end{aligned}
$$

We introduce a constant $q$ by setting $a_{0} a_{1} a_{2}=q$. Noting that the product $f_{0} f_{1} f_{2}$ can be regarded as the independent variable, we introduce a variable $c$ such that $f_{0} f_{1} f_{2}=q c^{2}$ and $\bar{c}=q c$. If we regard $f_{j}(j=0,1,2)$ as functions in $c$, eq.(11) thus represents a system of $q$-difference equations for the unknown functions $f_{j}=f_{j}(c)$ $(j=0,1,2)$ with parameters $a_{j}(j=0,1,2)$ such that $a_{0} a_{1} a_{2}=q$. As we will see in Subsection 1.3 below, this $q$-difference system has a continuous limit to the symmetric form of the fourth Painlevé equation $P_{\mathrm{IV}}$. For this reason, we refer to eq.(1) as the symmetric form of the fourth $q$-Painlevé equation $q \mathrm{P}_{\mathrm{IV}}$.

\subsection{Bäcklund transformations}

The discrete system eq.(11) admits the action of the (extended) affine Weyl group $\widetilde{W}=\left\langle s_{0}, s_{1}, s_{2}, \pi\right\rangle$ of type $A_{2}^{(1)}$ as a group of Bäcklund transformations. In what follows we denote by $\widetilde{W}$ the group generate by the generators $s_{0}, s_{1}, s_{2}, \pi$ and the the fundamental relations

$$
s_{i}^{2}=1, \quad\left(s_{i} s_{i+1}\right)^{3}=1, \quad \pi^{3}=1, \quad \pi s_{i}=s_{i+1} \pi \quad(i=0,1,2),
$$

where the indices are understood as elements of $\mathbb{Z} / 3 \mathbb{Z}$.

We define the action of $s_{0}, s_{1}, s_{2}, \pi$ on the parameters $a_{0}, a_{1}, a_{2}$ by the following formulas:

$$
\begin{array}{cccc}
s_{0}\left(a_{0}\right)=a_{0}^{-1}, & s_{0}\left(a_{1}\right)=a_{1} a_{0}, & s_{0}\left(a_{2}\right)=a_{2} a_{0}, \\
s_{1}\left(a_{0}\right)=a_{0} a_{1}, & s_{1}\left(a_{1}\right)=a_{1}^{-1}, & s_{1}\left(a_{2}\right)=a_{2} a_{1}, \\
s_{2}\left(a_{0}\right)=a_{0} a_{2}, & s_{2}\left(a_{1}\right)=a_{1} a_{2}, & s_{2}\left(a_{2}\right)=a_{2}^{-1}, \\
\pi\left(a_{0}\right)=a_{1}, & \pi\left(a_{1}\right)=a_{2}, & \pi\left(a_{2}\right)=a_{0} .
\end{array}
$$

Note that this definition is a multiplicative version of the standard realization of $\widetilde{W}$ on the simple roots $\alpha_{j}$ defined through $a_{j}=q^{\alpha_{j}}(j=0,1,2)$. We now define the action of $s_{0}, s_{1}, s_{2}, \pi$ on the $f$ variables as follows:

$$
\begin{aligned}
& s_{0}\left(f_{0}\right)=f_{0}, \quad s_{0}\left(f_{1}\right)=f_{1} \frac{a_{0}+f_{0}}{1+a_{0} f_{0}}, \quad s_{0}\left(f_{2}\right)=f_{2} \frac{1+a_{0} f_{0}}{a_{0}+f_{0}}, \\
& s_{1}\left(f_{0}\right)=f_{0} \frac{1+a_{1} f_{1}}{a_{1}+f_{1}}, \quad s_{1}\left(f_{1}\right)=f_{1}, \quad s_{1}\left(f_{2}\right)=f_{2} \frac{a_{1}+f_{1}}{1+a_{1} f_{1}}, \\
& s_{2}\left(f_{0}\right)=f_{0} \frac{a_{2}+f_{2}}{1+a_{2} f_{2}}, \quad s_{2}\left(f_{1}\right)=f_{1} \frac{1+a_{2} f_{2}}{a_{2}+f_{2}}, \quad s_{2}\left(f_{2}\right)=f_{2}, \\
& \pi\left(f_{0}\right)=f_{1}, \quad \pi\left(f_{1}\right)=f_{2}, \quad \pi\left(f_{2}\right)=f_{0} .
\end{aligned}
$$


We remark that the action of $s_{i}$ on the $a$-variables and the $f$-variables is given by

$$
s_{i}\left(a_{j}\right)=a_{j} a_{i}^{-a_{i j}}, \quad s_{i}\left(f_{j}\right)=f_{j}\left(\frac{a_{i}+f_{i}}{1+a_{i} f_{i}}\right)^{u_{i j}} \quad(i, j=0,1,2),
$$

respectively, where $A=\left(a_{i j}\right)_{i, j=0}^{2}$ is the generalized Cartan matrix of type $A_{2}^{(1)}$ and $U=\left(u_{i j}\right)_{i, j=0}^{2}$ is an orientation matrix of the corresponding Dynkin diagram:

$$
A=\left[\begin{array}{ccc}
2 & -1 & -1 \\
-1 & 2 & -1 \\
-1 & -1 & 2
\end{array}\right], \quad U=\left[\begin{array}{ccc}
0 & 1 & -1 \\
-1 & 0 & 1 \\
1 & -1 & 0
\end{array}\right]
$$

The following theorem can be verified by direct computation.

Theorem 1.1 The transformations $s_{0}, s_{1}, s_{2}$ and $\pi$ of the a-variables and the $f$-variables, defined by (何), (5), generate the extended affine Weyl group $\widetilde{W}=\left\langle s_{0}, s_{1}, s_{2}, \pi\right\rangle$ of type $A_{2}^{(1)}$. Furthermore, they commute with the time evolution $t$ of the fourth $q$-Painlevé equation $q P_{I V}$.

By using the action of the extended affine Weyl group $\widetilde{W}$, we can define the Schlesinger transformations $T_{1}$, $T_{2}, T_{3}$ for $q \mathrm{P}_{\mathrm{IV}}$ as

$$
T_{1}=\pi s_{2} s_{1}, \quad T_{2}=s_{1} \pi s_{2}, \quad T_{3}=s_{2} s_{1} \pi .
$$

Note that $T_{i} T_{j}=T_{j} T_{i}(i, j=1,2,3)$ and $T_{1} T_{2} T_{3}=1$. The action of $T_{1}$ on the variables $a_{j}, f_{j}$ is given explicitly as follows:

$$
\begin{aligned}
& T_{1}\left(a_{0}\right)=q a_{0}, \quad T_{1}\left(a_{1}\right)=q^{-1} a_{1}, \quad T_{1}\left(a_{2}\right)=a_{2}, \\
& T_{1}\left(f_{0}\right)=f_{1} \frac{\left(a_{0}+f_{0}\right)\left(a_{0}+f_{0}+a_{0} a_{2} f_{2}+a_{0}^{2} a_{2} f_{0} f_{2}\right)}{\left(1+a_{0} f_{0}\right)\left(a_{0}^{2} a_{2}+a_{0} a_{2} f_{0}+f_{2}+a_{0} f_{0} f_{2}\right)}, \\
& T_{1}\left(f_{1}\right)=f_{2} \frac{1+a_{0} f_{0}}{a_{0}+f_{0}}, \\
& T_{1}\left(f_{2}\right)=f_{0} \frac{a_{0}^{2} a_{2}+a_{0} a_{2} f_{0}+f_{2}+a_{0} f_{0} f_{2}}{a_{0}+f_{0}+a_{0} a_{2} f_{2}+a_{0}^{2} a_{2} f_{0} f_{2}} .
\end{aligned}
$$

The corresponding formulas for $T_{2}$ and $T_{3}$ are obtained by the rotation of indices, since $T_{2}=\pi T_{1} \pi^{-1}$ and $T_{3}=\pi T_{2} \pi^{-1}$. Each of these Schlesinger transformations commutes with the time evolution of $q \mathrm{P}_{\mathrm{IV}}$ and can be regarded as a version of the third $q$-Painlevé equation. For example, eq.(9) is rewritten equivalently as

$$
\begin{aligned}
& T_{1}\left(f_{1}\right)=f_{2} \frac{1+a_{0} f_{0}}{a_{0}+f_{0}}=\frac{q c^{2}}{f_{0} f_{1}} \frac{1+a_{0} f_{0}}{a_{0}+f_{0}}, \\
& T_{1}^{-1}\left(f_{0}\right)=f_{2} \frac{a_{1}+f_{1}}{1+a_{1} f_{1}}=\frac{q c^{2}}{f_{0} f_{1}} \frac{a_{1}+f_{1}}{1+a_{1} f_{1}} .
\end{aligned}
$$

This is an analogue of the fact that some discrete Painleve equations arise from Bäcklund transformations of the Painlevé equations $3,6,6,8,8,12$.

\subsection{Limit transition to $P_{\mathrm{IV}}$}

By introducing a small parameter $\varepsilon$ such that $q=e^{-\varepsilon^{2} / 2}$, we set

$$
a_{i}=e^{-\varepsilon^{2} \alpha_{i} / 2}, \quad f_{i}=-e^{-\varepsilon \varphi_{i}} \quad(j=0,1,2) .
$$

Then we have

$$
\overline{f_{i}}-f_{i}=\varepsilon^{2}\left(\varphi_{i}\left(\varphi_{i+1}-\varphi_{i+2}\right)+\alpha_{i}\right)+O\left(\varepsilon^{3}\right)
$$

for $i=0,1,2$. Passing to the variables $\alpha_{j}$ and $\varphi_{j}$, we define the derivation ' by

$$
x^{\prime}=\lim _{\varepsilon \rightarrow 0} \frac{1}{\varepsilon}(\bar{x}-x)
$$


for a function $x$ in $\alpha_{j}$ and $\varphi_{j}$. Then we have $\overline{f_{i}}-f_{i}=\varepsilon^{2} \varphi_{i}^{\prime}+O\left(\varepsilon^{3}\right)$. Hence we obtain

$$
\begin{aligned}
& \alpha_{0}^{\prime}=0, \quad \alpha_{1}^{\prime}=0, \quad \alpha_{2}^{\prime}=0, \\
& \varphi_{0}^{\prime}=\varphi_{0}\left(\varphi_{1}-\varphi_{2}\right)+\alpha_{0}, \\
& \varphi_{1}^{\prime}=\varphi_{1}\left(\varphi_{2}-\varphi_{0}\right)+\alpha_{1}, \\
& \varphi_{2}^{\prime}=\varphi_{2}\left(\varphi_{3}-\varphi_{1}\right)+\alpha_{2} .
\end{aligned}
$$

This differential system is the symmetric form the fourth Painlevé equation $P_{\mathrm{IV}}$ [19, 1, 14]. In fact, under the normalization $\varphi_{0}+\varphi_{1}+\varphi_{2}=t, \alpha_{0}+\alpha_{1}+\alpha_{2}=1$, the system (14) is equivalent to the second order differential equation

$$
y^{\prime \prime}=\frac{1}{2 y}\left(y^{\prime}\right)^{2}+\frac{3}{2} y^{3}-2 t y^{2}+\left(\frac{t^{2}}{2}+\alpha_{1}-\alpha_{2}\right) y-\frac{\alpha_{0}^{2}}{2 y}
$$

for $y=\varphi_{0}$, where ${ }^{\prime}=d / d t$. Through the limiting procedure, the Bäcklund transformations $s_{0}, s_{1}, s_{2}, \pi$ for our $q \mathrm{P}_{\mathrm{IV}}$ also pass to those for the symmetric form of $P_{\mathrm{IV}}$ (14) such that

$$
s_{i}\left(\alpha_{j}\right)=\alpha_{j}-\alpha_{i} a_{i j}, \quad s_{i}\left(\varphi_{j}\right)=\varphi_{j}+\frac{\alpha_{i}}{\varphi_{i}} u_{i j}, \quad(i, j=0,1,2)
$$

as in $[13,14]$.

\section{Particular solutions for $q \mathbf{P}_{\mathrm{IV}}$}

In this section, we investigate $q$-analogue of classical solutions of $\mathrm{P}_{\mathrm{IV}}$ and determinant formulas for them. Recall that $\mathrm{P}_{\mathrm{IV}}$ has two classes of classical solutions [15, 10, 14], those of hypergeometric type, expressed in terms of Hermite-Weber functions and the rational solutions.

\subsection{Continuous $q$-Hermite-Weber functions as seed solutions}

In order to show the parameter dependence explicitly, we rewrite $q \mathrm{P}_{\mathrm{IV}}$ (11) by operating $T_{1}^{\nu} T_{2}^{N}$ on (11) $(\nu, N \in \mathbb{Z})$. We denote $T_{1}^{\nu} T_{2}^{N}\left(f_{i}\right)=f_{i}(c ; \nu, N)$, and we abbreviate the unnecessary arguments depending on the context. Notice that

$$
T_{1}^{\nu} T_{2}^{N}\left(a_{0}\right)=a_{0} q^{\nu}, \quad T_{1}^{\nu} T_{2}^{N}\left(a_{1}\right)=a_{1} q^{-\nu+N}, \quad T_{1}^{\nu} T_{2}^{N}\left(a_{2}\right)=a_{2} q^{-N}, \quad T_{1}^{\nu} T_{2}^{N}(c)=c,
$$

and

$$
t\left(a_{i}\right)=a_{i},(i=0,1,2), \quad t(c)=q c,
$$

where

$$
q=a_{0} a_{1} a_{2}, \quad c=\left(\frac{f_{0} f_{1} f_{2}}{q}\right)^{1 / 2} .
$$

The $q$-Painlevé IV equation $q \mathrm{P}_{\mathrm{IV}}$ with respect to the variable $c$ is expressed as

$$
\begin{aligned}
& f_{0}(q c)=a_{0} a_{1} q^{N} f_{1}(c) \frac{1+a_{2} q^{-N} f_{2}(c)+a_{2} a_{0} q^{\nu-N} f_{2}(c) f_{0}(c)}{1+a_{0} q^{\nu} f_{0}(c)+a_{0} a_{1} q^{N} f_{0}(c) f_{1}(c)} \\
& f_{1}(q c)=a_{1} a_{2} q^{-\nu} f_{2}(c) \frac{1+a_{0} q^{\nu} f_{0}(c)+a_{0} a_{1} q^{N} f_{0}(c) f_{1}(c)}{1+a_{1} q^{-\nu+N} f_{1}(c)+a_{1} a_{2} q^{-\nu} f_{1}(c) f_{2}(c)}, \\
& f_{2}(q c)=a_{2} a_{0} q^{\nu-N} f_{0}(c) \frac{1+a_{1} q^{-\nu+N} f_{1}(c)+a_{1} a_{2} q^{-\nu} f_{1}(c) f_{2}(c)}{1+a_{2} q^{-N} f_{2}(c)+a_{2} a_{0} q^{\nu-N} f_{2}(c) f_{0}(c)} .
\end{aligned}
$$

where the arguments $\nu, N$ for $f_{i}$ are suppressed so that $f_{i}(c)=f_{i}(c ; \nu, N)$. In what follows, we use similar abbreviations

$$
f_{i}\left(q^{k} c\right)=f_{i}\left(q^{k} c ; \nu, N\right), \quad f_{i}(\nu+k)=f_{i}(c ; \nu+k, N), \quad f_{i}(N+k)=f_{i}(c ; \nu, N+k)
$$


for $k \in \mathbb{Z}$.

First let us consider the case $N=0$. It is possible to specialize the variables as

$$
f_{2}=-1, \quad a_{2}=1,
$$

consistently. In fact, eq.(22) becomes trivial by this specialization, and eqs.(20) and (21) are reduced to a discrete Riccati type equation

$$
f_{1}(q c)=-\frac{q}{a_{0} q^{\nu}} \frac{\left(1-q^{2} c^{2}\right) f_{1}(c)-a_{0} q^{\nu} c^{2}}{f_{1}(c)}
$$

and $f_{0}(c)=-\frac{q c^{2}}{f_{1}(c)}$. By putting $f_{1}(c)=\frac{F_{\nu}(c)}{G_{\nu}(c)}$ in eq.(25), we can solve eq. (25) as

$$
f_{1}(c)=-\frac{q}{a_{0} q^{\nu}} \frac{G_{\nu}(q c)}{G_{\nu}(c)},
$$

with $G_{\nu}(c)$ satisfying the linear $q$-difference equation

$$
G_{\nu}\left(q^{2} c\right)=\left(1-q^{2} c^{2}\right) G_{\nu}(q c)+a_{0}^{2} q^{2 \nu} c^{2} G_{\nu}(c) .
$$

Let us derive contiguity relations to be satisfied by $G_{\nu}(c)$ in the direction of $\nu$. For this purpose, we consider the Schlesinger transformation $T_{1}$ (9),

$$
\begin{aligned}
f_{0}(\nu+1)= & f_{1}(\nu) \frac{a_{0} q^{\nu}+f_{0}(\nu)}{1+a_{0} q^{\nu} f_{0}(\nu)} \\
& \times \frac{a_{0} q^{\nu}+f_{0}(\nu)+a_{0} a_{2} q^{\nu-N} f_{2}(\nu)+a_{0}^{2} a_{2} q^{2 \nu-N} f_{0}(\nu) f_{2}(\nu)}{a_{0}^{2} a_{2} q^{2 \nu-N}+a_{0} a_{2} q^{\nu-N} f_{0}(\nu)+f_{2}(\nu)+a_{0} q^{\nu} f_{0}(\nu) f_{2}(\nu)}, \\
f_{1}(\nu+1)= & f_{2}(\nu) \frac{1+a_{0} q^{\nu} f_{0}(\nu)}{a_{0} q^{\nu}+f_{0}(\nu)}, \\
f_{2}(\nu+1)= & f_{0}(\nu) \\
& \times \frac{a_{0}^{2} a_{2} q^{2 \nu-N}+a_{0} a_{2} q^{\nu-N} f_{0}(\nu)+f_{2}(\nu)+a_{0} q^{\nu} f_{0}(\nu) f_{2}(\nu)}{a_{0} q^{\nu}+f_{0}(\nu)+a_{0} a_{2} q^{\nu-N} f_{2}(\nu)+a_{0}^{2} a_{2} q^{2 \nu-N} f_{0}(\nu) f_{2}(\nu)} .
\end{aligned}
$$

Applying the specialization (24) and putting $N=0$, eqs.(28)-(30) are reduced to a discrete Riccati type equation,

$$
f_{1}(\nu+1)=-\frac{f_{1}(\nu)-a_{0} q^{\nu+1} c^{2}}{a_{0} q^{\nu} f_{1}(\nu)-q c^{2}}
$$

Substituting eq.26) into eq.(31), we obtain contiguity relations for $G_{\nu}(c)$,

$$
\begin{aligned}
& G_{\nu+1}(q c)=G_{\nu}(q c)+a_{0}^{2} q^{2 \nu} c^{2} G_{\nu}(c), \\
& G_{\nu+1}(c)=G_{\nu}(q c)+c^{2} G_{\nu}(c) .
\end{aligned}
$$

In particular, we obtain a three-term relation in the direction of $\nu$,

$$
G_{\nu+2}(c)=\left(c^{2}+1\right) G_{\nu+1}(c)-c^{2}\left(1-a_{0}^{2} q^{2 \nu}\right) G_{\nu}(c) .
$$

We note that eqs. (27) and (34) are derived from eqs.(32) and (33).

From the above discussion, we have:

Proposition $2.1 q P_{I V}$ (20)-29) admits 1-parameter family of particular solutions given by

$$
f_{0}=c^{2} a_{0} q^{\nu} \frac{G_{\nu}(c)}{G_{\nu}(q c)}, \quad f_{1}=-\frac{q}{a_{0} q^{\nu}} \frac{G_{\nu}(q c)}{G_{\nu}(c)}, \quad f_{2}=-1
$$

for $N=0$, where $G_{\nu}(c)$ is a function satisfying the contiguity relations (38) and (33). 
It is interesting to note that eq.(27) admits polynomial solutions in $c$ if $a_{0}=q$,

$$
\begin{aligned}
G_{0}(c) & =1 \\
G_{1}(c) & =c^{2}+1 \\
G_{2}(c) & =c^{4}+\left(1+q^{2}\right) c^{2}+1, \\
G_{3}(c) & =c^{6}+\left(1+q^{2}+q^{4}\right) c^{4}+\left(1+q^{2}+q^{4}\right) c^{2}+1, \\
& \cdots
\end{aligned}
$$

It is not difficult to check that

$$
G_{\nu}(c)=\sum_{k=0}^{\nu} \frac{\left(q^{2} ; q^{2}\right)_{\nu}}{\left(q^{2} ; q^{2}\right)_{k}\left(q^{2} ; q^{2}\right)_{\nu-k}} c^{2 \nu-2 k}=\sum_{k=0}^{\nu}\left[\begin{array}{l}
\nu \\
k
\end{array}\right]_{q^{2}} c^{2 \nu-2 k} \quad(\nu=0,1,2, \cdots)
$$

satisfy eqs. (32) and (33), where $\left[\begin{array}{l}\nu \\ k\end{array}\right]_{q}$ is the $q$-binomial coefficient. The generating function for these polynomials is given by

$$
\frac{1}{\left(c \lambda ; q^{2}\right)\left(\lambda / c ; q^{2}\right)}=\sum_{\nu=0}^{\infty} \frac{G_{\nu}(c)}{\left(q^{2} ; q^{2}\right)_{\nu}}\left(\frac{\lambda}{c}\right)^{\nu} .
$$

The polynomials $H_{\nu}(x)=c^{-\nu} G_{\nu}(c), x=\frac{c+c^{-1}}{2}$ are called the continuous $q$-Hermite polynomials [1], 2]. In this sense, $c^{-\nu} G_{\nu}(c)$ for $a_{0} \neq q^{l}(l \in \mathbb{Z})$ may be regarded as a $q$-difference analogue of the Hermite-Weber functions, to which hereafter we will refer as "continuous $q$-Hermite-Weber functions".

We note that it is possible to obtain "higher order" solutions for $N \in \mathbb{Z}$ by successive applications of $T_{2}$ on the solutions obtained abovel. These solutions are expressed rationally in the continuous $q$-Hermite-Weber functions.

From the rotational symmetry $\pi$ of $q \mathrm{P}_{\mathrm{IV}}$, it is possible to apply other specialization, $\left(a_{0}, f_{0}\right)=(1,-1)$, $\nu=0$ or $\left(a_{1}, f_{1}\right)=(1,-1), \nu-N=0$ on $q \mathrm{P}_{\mathrm{IV}}(20)-22$ and perform the same procedure as discussed above. Therefore, we obtain

Theorem 2.2 When $a_{i}=q^{k}$ for some $i=0,1,2$ and $k \in \mathbb{Z}$, the fourth $q$-Painlevé equation $q P_{I V}$ (1) admits a 1-parameter family of particular solutions which are expressed rationally by the continuous q-Hermite-Weber functions.

Explicit description of these solutions will be given in the next section.

Note that the hypersurfaces $a_{i}=q^{k}(i=0,1,2 ; k \in \mathbb{Z})$ in the parameter space correspond to the reflection hyperplanes of the affine Weyl group $W=W\left(A_{2}^{(1)}\right)$. This is an analogue of a well-known result by Okamoto for the classical solutions of the continuous $\mathrm{P}_{\mathrm{IV}}[15$.

\subsection{Toeplitz determinants}

\subsubsection{Determinant formula and bilinear equations}

In this section, we prove the following determinant formula for the particular solutions obtained in the previous section.

Theorem 2.3 Let $G_{\nu}(c)$ is a solution of eqs. 39) and (33). For each $N \in \mathbb{Z}_{\geq 0}$, we define an $N \times N$ Toeplitz determinant

$$
\phi_{N}^{\nu}(c)=\operatorname{det}\left(G_{\nu-i+j}(c)\right)_{i, j=1, \cdots, N} \quad\left(N \in \mathbb{Z}_{>0}\right), \quad \phi_{0}^{\nu}(c)=1 .
$$

Then,

$$
\begin{aligned}
& f_{0}(c ; \nu, N)=a_{0} q^{\nu} c^{2} \frac{\phi_{N+1}^{\nu}(c) \phi_{N}^{\nu}(q c)}{\phi_{N+1}^{\nu}(q c) \phi_{N}^{\nu}(c)} \\
& f_{1}(c ; \nu, N)=-\frac{1}{a_{0} q^{\nu+N-1}} \frac{\phi_{N+1}^{\nu}(q c) \phi_{N}^{\nu-1}(c)}{\phi_{N+1}^{\nu}(c) \phi_{N}^{\nu-1}(q c)}, \\
& f_{2}(c ; \nu, N)=-q^{N} \frac{\phi_{N}^{\nu-1}(q c) \phi_{N}^{\nu}(c)}{\phi_{N}^{\nu}(q c) \phi_{N}^{\nu-1}(c)}
\end{aligned}
$$

\footnotetext{
${ }^{1}$ The situation is a little delicate for $N<0$, but we will discuss in Section 2.2.3.
} 
satisfy $q P_{I V}$ (20)-(29) with $a_{2}=1$.

We note that the case of $N=0$ agrees with Proposition 2.1.

Theorem 2.3 is a direct consequence of the following proposition:

Proposition $2.4 \phi_{N}^{\nu}(c)$ satisfies the following bilinear difference equations,

$$
\begin{aligned}
& a_{0}^{2} c^{2} q^{2 \nu} \phi_{N}^{\nu}(q c) \phi_{N+1}^{\nu}(c)+\phi_{N}^{\nu}(c) \phi_{N+1}^{\nu}(q c)=q^{-2 N} \phi_{N+1}^{\nu+1}(q c) \phi_{N}^{\nu-1}(c), \\
& a_{0}^{2} q^{2 \nu} \phi_{N}^{\nu}(q c) \phi_{N+1}^{\nu+1}(c)-\phi_{N}^{\nu}(c) \phi_{N+1}^{\nu+1}(q c)=\left(a_{0}^{2} q^{2 \nu}-q^{2 N}\right) \phi_{N+1}^{\nu}(q c) \phi_{N}^{\nu+1}(c), \\
& \phi_{N+1}^{\nu+1}(q c) \phi_{N}^{\nu}(c)-q^{2 N} \phi_{N+1}^{\nu+1}(c) \phi_{N}^{\nu}(q c)=c^{2}\left(a_{0}^{2} q^{2 \nu}-q^{2 N}\right) \phi_{N+1}^{\nu}(c) \phi_{N}^{\nu+1}(q c), \\
& \phi_{N}^{\nu+1}(q c) \phi_{N}^{\nu}(c)-\phi_{N}^{\nu+1}(c) \phi_{N}^{\nu}(q c)=-\phi_{N-1}^{\nu}(q c) \phi_{N+1}^{\nu+1}(c), \\
& \phi_{N}^{\nu+1}(q c) \phi_{N}^{\nu}(c)-q^{2 N} \phi_{N}^{\nu+1}(c) \phi_{N}^{\nu}(q c)=\frac{1}{a_{0}^{2} q^{2 \nu} c^{2}} \phi_{N+1}^{\nu+1}(q c) \phi_{N-1}^{\nu}(c), \\
& q^{2 N} \phi_{N}^{\nu}(c) \phi_{N+1}^{\nu}(c)-\phi_{N+1}^{\nu+1}(c) \phi_{N}^{\nu-1}(c)=-a_{0}^{2} c^{2} q^{2(\nu-1)} \phi_{N+1}^{\nu}\left(q^{-1} c\right) \phi_{N}^{\nu}(q c), \\
& \phi_{N}^{\nu}(c) \phi_{N+1}^{\nu+1}(c)+c^{2}\left(a_{0}^{2} q^{2 \nu}-q^{2 N}\right) \phi_{N+1}^{\nu}(c) \phi_{N}^{\nu+1}(c)=q^{-2 N} \phi_{N}^{\nu}\left(q^{-1} c\right) \phi_{N+1}^{\nu+1}(q c), \\
& a_{0}^{2} q^{2 \nu} \phi_{N}^{\nu}(c) \phi_{N}^{\nu+1}(c)+\phi_{N+1}^{\nu+1} \phi_{N-1}^{\nu}(c)=a_{0}^{2} q^{2 \nu} \phi_{N}^{\nu+1}\left(q^{-1} c\right) \phi_{N}^{\nu}(q c) .
\end{aligned}
$$

Proof of Theorem 2.3. We show that $f_{i}(c)(i=0,1,2)$ defined by eqs.(39)-(41) satisfy eq.(20). Substituting eqs.(39)-(41) into the numerator of right hand side of eq.(20), we have

$$
\begin{aligned}
& 1+q^{-N} f_{2}(c)+a_{0} q^{\nu-N} f_{2}(c) f_{0}(c)=1+q^{-N} f_{2}(c)\left(1+a_{0}^{2} q^{2 \nu} c^{2} \frac{\phi_{N+1}^{\nu}(c) \phi_{N}^{\nu}(q c)}{\phi_{N+1}^{\nu}(q c) \phi_{N}^{\nu}(c)}\right) \\
= & 1+q^{-N} f_{2}(c) \frac{\phi_{N+1}^{\nu}(q c) \phi_{N}^{\nu}(c)+a_{0}^{2} q^{2 \nu} c^{2} \phi_{N+1}^{\nu}(c) \phi_{N}^{\nu}(q c)}{\phi_{N+1}^{\nu}(q c) \phi_{N}^{\nu}(c)} \\
= & 1+q^{-N} f_{2}(c) q^{-2 N} \frac{\phi_{N+1}^{\nu+1}(q c) \phi_{N}^{\nu-1}(c)}{\phi_{N+1}^{\nu}(q c) \phi_{N}^{\nu}(c)}=1-q^{-2 N} \frac{\phi_{N}^{\nu-1}(q c) \phi_{N+1}^{\nu+1}(q c)}{\phi_{N}^{\nu}(q c) \phi_{N+1}^{\nu}(q c)} \\
= & \frac{\phi_{N}^{\nu}(q c) \phi_{N+1}^{\nu}(q c)-q^{-2 N} \phi_{N}^{\nu-1}(q c) \phi_{N+1}^{\nu+1}(q c)}{\phi_{N}^{\nu}(q c) \phi_{N+1}^{\nu}(q c)}=-a_{0}^{2} c^{2} q^{2(\nu-N)} \frac{\phi_{N+1}^{\nu}(c) \phi_{N}^{\nu}\left(q^{2} c\right)}{\phi_{N}^{\nu}(q c) \phi_{N+1}^{\nu}(q c)},
\end{aligned}
$$

where we used bilinear equations (42) and (47). The denominator is calculated by using eq.(43) and (48) as

$$
1+a_{0} q^{\nu} f_{0}(c)+q^{N+1} f_{0}(c) f_{1}(c)=\frac{q^{-2 N} \phi_{N}^{\nu-1}(c) \phi_{N+1}^{\nu}\left(q^{2} c\right)}{\phi_{N+1}^{\nu}(q c) \phi_{N}^{\nu-1}(q c)} .
$$

Then, the right hand side of eq.(20) reduces to

$$
\begin{aligned}
& q^{N+1} f_{1}(c) \frac{1+q^{-N} f_{2}(c)+a_{0} q^{\nu-N} f_{2}(c) f_{0}(c)}{1+a_{0} q^{\nu} f_{0}(c)+q^{N+1} f_{0}(c) f_{1}(c)} \\
= & q^{N+1} \cdot \frac{1}{a_{0} q^{\nu+N-1}} \frac{\phi_{N+1}^{\nu}(q c) \phi_{N}^{\nu-1}(c)}{\phi_{N+1}^{\nu}(c) \phi_{N}^{\nu-1}(q c)} \cdot a_{0}^{2} c^{2} q^{2(\nu-N)} \frac{\phi_{N+1}^{\nu}(c) \phi_{N}^{\nu}\left(q^{2} c\right)}{\phi_{N}^{\nu}(q c) \phi_{N+1}^{\nu}(q c)} \cdot q^{2 N} \frac{\phi_{N+1}^{\nu}(q c) \phi_{N}^{\nu-1}(q c)}{\phi_{N}^{\nu-1}(c) \phi_{N+1}^{\nu}\left(q^{2} c\right)} \\
= & a_{0} c^{2} q^{\nu+2} \frac{\phi_{N+1}^{\nu}(q c) \phi_{N}^{\nu}\left(q^{2} c\right)}{\phi_{N+1}^{\nu}\left(q^{2} c\right) \phi_{N}^{\nu}(q c)}=f_{0}(q c) .
\end{aligned}
$$

Thus we have shown that eq.(20) follows. Eq.(21) is checked by using the bilinear equations (43), (47), (45) and(49). Eq.(22) follows automatically.

Remark. It should be noted that both $1+a_{i} f_{i}$ and $1+f_{i} / a_{i}(i=0,1,2)$ admit multiplicative formula with respect to $\phi$. In fact, we have,

$$
1+a_{0} q^{\nu} f_{0}(c ; \nu, N)=q^{-2 N} \frac{\phi_{N+1}^{\nu+1}(q c) \phi_{N}^{\nu-1}(c)}{\phi_{N+1}^{\nu}(q c) \phi_{N}^{\nu}(c)}
$$




$$
\begin{aligned}
& 1+a_{1} q^{-\nu+N} f_{1}(c ; \nu, N)=\frac{a_{0}^{2} q^{2(\nu-1)}-q^{2 N}}{a_{0}^{2} q^{2(\nu-1)}} \frac{\phi_{N+1}^{\nu-1}(q c) \phi_{N}^{\nu}(c)}{\phi_{N+1}^{\nu}(c) \phi_{N}^{\nu-1}(q c)} \\
& 1+a_{2} q^{-N} f_{2}(c ; \nu, N)=-\frac{\phi_{N-1}^{\nu-1}(q c) \phi_{N+1}^{\nu}(c)}{\phi_{N}^{\nu-1}(c) \phi_{N}^{\nu}(q c)}
\end{aligned}
$$

and

$$
\begin{aligned}
& 1+\frac{f_{0}(c ; \nu, N)}{a_{0} q^{\nu}}=\frac{\phi_{N}^{\nu-1}(q c) \phi_{N+1}^{\nu+1}(c)}{\phi_{N+1}^{\nu}(q c) \phi_{N}^{\nu}(c)} \\
& 1+\frac{f_{1}(c ; \nu, N)}{a_{1} q^{-\nu+N}}=q^{-2 N} c^{2}\left(q^{2 N}-a_{0}^{2} q^{2(\nu-1)}\right) \frac{\phi_{N+1}^{\nu-1}(c) \phi_{N}^{\nu}(q c)}{\phi_{N+1}^{\nu}(c) \phi_{N}^{\nu-1}(q c)} \\
& 1+\frac{f_{2}(c ; \nu, N)}{a_{2} q^{-N}}=\frac{1}{a_{0}^{2} c^{2} q^{2(\nu-1)}} \frac{\phi_{N+1}^{\nu}(q c) \phi_{N-1}^{\nu-1}(c)}{\phi_{N}^{\nu}(q c) \phi_{N}^{\nu-1}(c)}
\end{aligned}
$$

which can be verified directly by using eqs.(39)-(41) and the bilinear equations (42)-(46).

\subsubsection{Proof of Proposition 2.4}

Our basic idea for proving Proposition 2.4 is as follows. Bilinear difference equations are derived from the Plücker relations, which are quadratic identities among determinants whose columns are shifted. Therefore, we first construct such "difference formulas" that relate "shifted determinants" and $\phi_{N}^{\nu}(c)$, by using the contiguity relations of $G_{\nu}(c)$. We then derive bilinear difference equations with the aid of difference formulas from proper Plücker relations. We take eq.(42) as an example to show this procedure explicitly. For other equations, see Appendix A.

Let us introduce notations,

$$
\phi_{N}^{\nu, m}=\left|\begin{array}{cccc}
G_{\nu}^{m}\left(q^{m} c\right)=G_{\nu}^{m}, \quad \phi_{N}^{\nu}\left(q^{m} c\right)=\phi_{N}^{\nu, m}, \\
G_{\nu-1}^{m} & G_{\nu+1}^{m} & \cdots & G_{\nu+N-1}^{m} \\
\vdots & \vdots & \ddots & \vdots \\
G_{\nu-N+1}^{m} & G_{\nu-N+2}^{m} & \cdots & G_{\nu}^{m}
\end{array}\right|=\left|\mathbf{0}^{m}, \mathbf{1}^{m}, \cdots, \mathbf{N}-\mathbf{1}^{m}\right|,
$$

where $\mathbf{k}^{m}$ denotes a column vector,

$$
\mathbf{k}^{m}=\left(\begin{array}{c}
G_{\nu+k}^{m} \\
G_{\nu+k-1}^{m} \\
\vdots \\
G_{\nu+k-N+1}^{m}
\end{array}\right)
$$

Here the height of the column is $N$, but we use the same symbol for determinants with different size, since there is no possibility of confusion.

We next construct a difference formula.

\section{Lemma 2.5 (Difference Formula I)}

$$
\begin{aligned}
& \left|\mathbf{0}^{m+1 \prime}, \mathbf{0}^{m}, \mathbf{1}^{m}, \cdots, \mathbf{N}-\mathbf{3}^{m}, \mathbf{N}-\mathbf{2}^{m}\right|=a_{0}^{-2(N-1)} c^{-2(N-1)} q^{-2(N-1)(m+\nu-1)} \phi_{N}^{\nu, m+1}, \\
& \left|\mathbf{1}^{m+1 \prime}, \mathbf{0}^{m}, \mathbf{1}^{m}, \cdots, \mathbf{N}-\mathbf{3}^{m}, \mathbf{N}-\mathbf{2}^{m}\right|=a_{0}^{-2(N-1)} c^{-2(N-1)} q^{-2(N-1)(m+\nu-1)} \phi_{N}^{\nu, m+1},
\end{aligned}
$$

where $\mathbf{k}^{m \prime}$ is a column vector,

$$
\mathbf{k}^{m \prime}=\left(\begin{array}{c}
G_{\nu+k}^{m} \\
q^{2} G_{\nu+k-1}^{m} \\
\vdots \\
q^{2(N-1)} G_{\nu+k-N+1}^{m}
\end{array}\right)
$$


Proof of Lemma 2.5. We use the contiguity relation (32), which is rewritten as

$$
G_{\nu+1}^{m+1}=G_{\nu}^{m+1}+a_{0}^{2} q^{2(\nu+m)} c^{2} G_{\nu}^{m} .
$$

in the present notation. Then we have

$$
\begin{aligned}
& \phi_{N}^{\nu, m+1}=\left|\begin{array}{cccc}
G_{\nu}^{m+1} & G_{\nu+1}^{m+1} & \cdots & G_{\nu+N-1}^{m+1} \\
G_{\nu-1}^{m+1} & G_{\nu}^{m+1} & \cdots & G_{\nu-N-2}^{m+1} \\
\vdots & \vdots & \ddots & \vdots \\
G_{\nu-N+1}^{m+1} & G_{\nu-N+2}^{m+1} & \cdots & G_{\nu}^{m+1}
\end{array}\right| \\
& =\left|\begin{array}{cccc}
G_{\nu}^{m+1} & G_{\nu+1}^{m+1}-G_{\nu+1}^{m+1} & \cdots & G_{\nu+N-1}^{m+1}-G_{\nu+N-2}^{m+1} \\
G_{\nu-1}^{m+1} & G_{\nu}^{m+1}-G_{\nu-1}^{m+1} & \cdots & G_{\nu+N-2}^{m+1}-G_{\nu+N-3}^{m+1} \\
\vdots & \vdots & \ddots & \vdots \\
G_{\nu-N+1}^{m+1} & G_{\nu-N+2}^{m+1}-G_{\nu-N+1}^{m+1} & \cdots & G_{\nu}^{m+1}-G_{\nu-1}^{m+1}
\end{array}\right| \\
& =\left|\begin{array}{cccc}
G_{\nu}^{m+1} & a_{0}^{2} c^{2} q^{2(\nu+m)} G_{\nu}^{m} & \cdots & a_{0}^{2} q^{2(\nu+N-2+m)} c^{2} G_{\nu+N-2}^{m} \\
G_{\nu-1}^{m+1} & a_{0}^{2} c^{2} q^{2(\nu-1+m)} G_{\nu-1}^{m} & \cdots & a_{0}^{2} q^{2(\nu+N-3+m)} c^{2} G_{\nu+N-3}^{m} \\
\vdots & \vdots & \ddots & \vdots \\
G_{\nu-N+1}^{m+1} & a_{0}^{2} c^{2} q^{2(\nu-N+1+m)} G_{\nu-N+1}^{m+1} & \cdots & a_{0}^{2} q^{2(\nu-1+m)} c^{2} G_{\nu-1}^{m}
\end{array}\right| \\
& =a_{0}^{2(N-1)} c^{2(N-1)} q^{2(N-1)(\nu+m-1)}\left|\begin{array}{cccc}
G_{\nu}^{m+1} & G_{\nu}^{m} & \cdots & G_{\nu+N-2}^{m} \\
q^{2} G_{\nu-1}^{m+1} & G_{\nu-1}^{m} & \cdots & G_{\nu+N-3}^{m} \\
\vdots & \vdots & \ddots & \vdots \\
q^{2(N-1)} G_{\nu-N+1}^{m} & G_{\nu-N+1}^{m} & \cdots & G_{\nu-1}^{m}
\end{array}\right| \\
& =a_{0}^{2(N-1)} c^{2(N-1)} q^{2(N-1)(\nu+m-1)}\left|\mathbf{0}^{m+1}, \mathbf{0}^{m}, \mathbf{1}^{m}, \cdots, \mathbf{N}-\mathbf{3}^{m}, \mathbf{N}-\mathbf{2}^{m}\right| \text {, }
\end{aligned}
$$

which is eq.(60). In the second line of the above calculation, adding the second column to the first column, we get

$$
\phi_{N}^{\nu, m+1}=\left|\begin{array}{cccc}
G_{\nu+1}^{m+1} & G_{\nu+1}^{m+1}-G_{\nu}^{m+1} & \cdots & G_{\nu+N-1}^{m+1}-G_{\nu+N-2}^{m+1} \\
G_{\nu}^{m+1} & G_{\nu}^{m+1}-G_{\nu-1}^{m+1} & \cdots & G_{\nu-N-2}^{m+1}-G_{\nu+N-3}^{m+1} \\
\vdots & \vdots & \ddots & \vdots \\
G_{\nu-N+2}^{m+1} & G_{\nu-N+2}^{m+1}-G_{\nu-N+1}^{m+1} & \cdots & G_{\nu}^{m+1}-G_{\nu-1}^{m+1}
\end{array}\right|,
$$

from which we obtain eq. (61) by using eq.(63).

We then consider the Plücker relation,

$$
\begin{aligned}
0 & =\left|\varphi_{1}, \mathbf{1}^{m+1 \prime}, \mathbf{1}^{m}, \cdots, \mathbf{N}-\mathbf{1}^{m}\right| \times\left|\mathbf{0}^{m}, \mathbf{1}^{m}, \cdots, \mathbf{N}-\mathbf{1}^{m}, \mathbf{N}^{m}\right| \\
& -\left|\varphi_{1}, \mathbf{0}^{m}, \mathbf{1}^{m}, \cdots, \mathbf{N}-\mathbf{1}^{m}\right| \times\left|\mathbf{1}^{m+1 \prime}, \mathbf{1}^{m}, \cdots, \mathbf{N}-\mathbf{1}^{m}, \mathbf{N}^{m}\right| \\
& +\left|\mathbf{1}^{m+1 \prime}, \mathbf{0}^{m}, \mathbf{1}^{m}, \cdots, \mathbf{N}-\mathbf{1}^{m}\right| \times\left|\varphi_{1}, \mathbf{1}^{m}, \cdots, \mathbf{N}-\mathbf{1}^{m}, \mathbf{N}^{m}\right|
\end{aligned}
$$

where

$$
\varphi_{1}=\left(\begin{array}{c}
1 \\
0 \\
\vdots \\
0
\end{array}\right)
$$

By expansion with respect to the column $\varphi_{1}$, the Plücker relation (65) is rewritten as

$$
\begin{aligned}
0 & =q^{2}\left|\mathbf{0}^{m+1 \prime}, \mathbf{0}^{m}, \cdots, \mathbf{N}-\mathbf{2}^{m}, \mathbf{N}-\mathbf{1}^{m}\right| \times\left|\mathbf{0}^{m}, \mathbf{1}^{m}, \cdots, \mathbf{N}-\mathbf{1}^{m}\right| \\
& -\left|-\mathbf{1}^{m}, \mathbf{0}^{m}, \cdots, \mathbf{N}-\mathbf{2}^{m}, \mathbf{N}-\mathbf{1}^{m}\right| \times\left|\mathbf{1}^{m+1 \prime}, \mathbf{1}^{m}, \cdots, \mathbf{N}-\mathbf{1}^{m}\right| \\
& +\left|\mathbf{1}^{m+1 \prime}, \mathbf{0}^{m}, \mathbf{1}^{m}, \cdots, \mathbf{N}-\mathbf{1}^{m}\right| \times\left|\mathbf{0}^{m}, \cdots, \mathbf{N}-\mathbf{2}^{m}, \mathbf{N}-\mathbf{1}^{m}\right|
\end{aligned}
$$


where we have used

$$
\begin{aligned}
& \left|\varphi_{1}, \mathbf{1}^{m+1 \prime}, \mathbf{1}^{m}, \cdots, \mathbf{N}-\mathbf{1}^{m}\right|=\left|\begin{array}{ccccc}
1 & G_{\nu+1}^{m+1} & G_{\nu+1}^{m} & \cdots & G_{\nu+N-1}^{m} \\
0 & q^{2} G_{\nu}^{m+1} & G_{\nu}^{m} & \cdots & G_{\nu+N-2}^{m} \\
\vdots & \vdots & \vdots & \ddots & \vdots \\
0 & q^{2 N} G_{\nu-N+1}^{m+1} & G_{\nu-N+2}^{m} & \cdots & G_{\nu-1}^{m}
\end{array}\right| \\
= & \left|\begin{array}{cccc}
q^{2} G_{\nu}^{m+1} & G_{\nu}^{m} & \cdots & G_{\nu+N-2}^{m} \\
\vdots & \vdots & \ddots & \vdots \\
q^{2 N} G_{\nu-N+1}^{m+1} & G_{\nu-N+2}^{m} & \cdots & G_{\nu-1}^{m}
\end{array}\right|=q^{2}\left|\mathbf{0}^{m+1 \prime}, \mathbf{0}^{m}, \cdots, \mathbf{N}-\mathbf{2}^{m}, \mathbf{N}-\mathbf{1}^{m}\right| .
\end{aligned}
$$

Then, we obtain by using Lemma 2.5,

$$
\begin{aligned}
0 & =q^{2} \times a_{0}^{-2(N-1)} c^{-2(N-1)} q^{-2(N-1)(\nu+m-1)} \phi_{N}^{\nu, m+1} \times \phi_{N+1}^{\nu, m} \\
& -\phi_{N}^{\nu-1, m} \times a_{0}^{-2 N} c^{-2 N} q^{-2 N(\nu+m)} \phi_{N+1}^{\nu+1, m+1} \\
& +a_{0}^{-2 N} c^{-2 N} q^{-2 N(\nu+m-1)} \phi_{N+1}^{\nu, m+1} \times \phi_{N}^{\nu, m},
\end{aligned}
$$

which is the same as eq.(42). This completes the proof of the bilinear equation (42).

\subsubsection{Determinant formula for negative $N$}

Proposition 2.3 is a determinant formula for the solutions with $N \in \mathbb{Z}_{\geq 0}$, which are obtained by successive application of $T_{2}$ on the seed solution described in Proposition 2.1. In order to obtain solutions for $N \in \mathbb{Z}_{<0}$, we have to apply $T_{2}^{-1}$ on the seed solution, however, we find that this procedure collapses. Therefore, we construct "seed and higher solutions" for negative $N$ so that bilinear equations described in Proposition 2.4 hold for all $N \in \mathbb{Z}_{<0}$. To find the seed solutions for $N=-1$, we put $N=-1, \phi_{-1}^{\nu}(c)=\bar{G}_{\nu}(c)$ and $\phi_{0}^{\nu}(c)=1$ in eqs.(42)-(49), we obtain a set of contiguity relations to be satisfied by $\bar{G}_{\nu}(c)$,

$$
\begin{aligned}
& a_{0}^{2} c^{2} q^{2 \nu} \bar{G}_{\nu}(q c)+\bar{G}_{\nu}(c)=q^{2} \bar{G}_{\nu-1}(c), \\
& \bar{G}_{\nu}(q c)=\bar{G}_{\nu+1}(c)+c^{2} \bar{G}_{\nu+1}(q c) .
\end{aligned}
$$

Similarly to the case of $N \geq 0$, the following three-term relations in the direction of $c$ or $\nu$ are derived from eqs. 68) and (69),

$$
\begin{aligned}
& \bar{G}_{\nu}(c)=\left(\frac{1}{q^{2}}-c^{2}\right) \bar{G}_{\nu}(q c)+a_{0}^{2} q^{2 \nu} c^{2} \bar{G}_{\nu}\left(q^{2} c\right), \\
& \bar{G}_{\nu}(c)=\frac{1}{q^{2}}\left(1+c^{2}\right) \bar{G}_{\nu+1}(c)-\frac{c^{2}}{q^{4}}\left(1-a_{0}^{2} q^{2 \nu}\right) \bar{G}_{\nu+2}(c) .
\end{aligned}
$$

Then we obtain a determinant formula for $N<0$ as follows.

Theorem 2.6 Let $\bar{G}_{\nu}(c)$ is a function satisfying eqs. (68) and (69). For each $-M=N \in \mathbb{Z}_{<0}$, we define an $M \times M$ Toeplitz determinant

$$
\phi_{N}^{\nu}(c)=\operatorname{det}\left(\bar{G}_{\nu-i+j}(c)\right)_{i, j=1, \cdots, M} \quad\left(-M=N \in \mathbb{Z}_{<0}\right),
$$

Then, $\phi_{N}^{\nu}(c)$ satisfies the bilinear equations (49)-(49), and therefore eqs.(39)-(41) satisfy $q P_{I V}(20)$-(28) with $a_{2}=1$.

Since this theorem is proved by similar procedure to Theorem 2.3, we omit the details.

Remark If we parametrize as $a_{0}=q^{\mu}$,

$$
\bar{G}_{\nu}(c ; q)=c^{2} q^{-2 \nu} G_{-(\nu-2 \mu)}(c ; 1 / q),
$$

satisfies eqs.(68) and (69) formally. 


\section{$2.3 q$-Okamoto polynomials}

In the previous sections we have discussed the particular solutions on the reflection hyperplane of the affine Weyl group $W=W\left(A_{2}^{(1)}\right)$ in the parameter space. It is easy to see that $q \mathrm{P}_{\mathrm{IV}}$ (1) with normalization condition (19) admits a particular solution given by

$$
\left(f_{0}, f_{1}, f_{2} ; a_{0}, a_{1}, a_{2}\right)=\left(x p^{-1}, x p^{-1}, x p^{-1} ; p^{-1}, p^{-1}, p^{-1}\right), \quad x=c^{2 / 3}, \quad p=q^{-1 / 3} .
$$

Note that this parameter corresponds to the fixed point of $\pi$, namely Dynkin diagram automorphism of the

affine Weyl group $W\left(A_{2}^{(1)}\right)$. By applying the Bäcklund transformations on this seed solution, we get a series of rational solutions on the barycenters of the Weyl chamber of the affine Weyl group $W\left(A_{2}^{(1)}\right)$.

Theorem 2.7 For the parameters

$$
T_{1}^{m} T_{2}^{n}\left(a_{0}, a_{1}, a_{2}\right)=\left(p^{-3 m-1}, p^{3 m-3 n-1}, p^{3 n-1}\right), \quad m, n \in \mathbb{Z},
$$

we have the following rational solutions of $q \mathrm{P}_{\mathrm{IV}}$ equation (4),

$$
\begin{aligned}
& T_{1}^{m} T_{2}^{n}\left(f_{0}\right)=x p^{m-2 n-1} \frac{Q_{m+1, n}\left(x p^{-2}\right) Q_{m+1, n+1}(x)}{Q_{m+1, n}(x) Q_{m+1, n+1}\left(x p^{-2}\right)}, \\
& T_{1}^{m} T_{2}^{m}\left(f_{1}\right)=x p^{m+n-1} \frac{Q_{m+1, n+1}\left(x p^{-2}\right) Q_{m, n}(x)}{Q_{m+1, n+1}(x) Q_{m, n}\left(x p^{-2}\right)} \\
& T_{1}^{m} T_{2}^{n}\left(f_{2}\right)=x p^{n-2 m-1} \frac{Q_{m, n}\left(x p^{-2}\right) Q_{m+1, n}(x)}{Q_{m, n}(x) Q_{m+1, n}\left(x p^{-2}\right)} .
\end{aligned}
$$

Here $Q_{m, n}(x)$ are monic polynomials defined through the recurrence relations,

$$
\begin{aligned}
Q_{m-1, n}(x) Q_{m+1, n+1}\left(x p^{-2}\right) & =Q_{m, n}(x) Q_{m, n+1}\left(x p^{-2}\right)+x p^{-2 m-2 n} Q_{m, n+1}(x) Q_{m, n}\left(x p^{-2}\right), \\
Q_{m+1, n}(x) Q_{m, n+1}\left(x p^{-2}\right) & =Q_{m+1, n+1}(x) Q_{m, n}\left(x p^{-2}\right)+x p^{4 m-2 n-2} Q_{m, n}(x) Q_{m+1, n+1}\left(x p^{-2}\right), \\
Q_{m+1, n+1}(x) Q_{m, n-1}\left(x p^{-2}\right) & =Q_{m, n}(x) Q_{m+1, n}\left(x p^{-2}\right)+x p^{4 n-2 m-2} Q_{m+1, n}(x) Q_{m, n}\left(x p^{-2}\right),
\end{aligned}
$$

with initial conditions $Q_{0,0}(x)=Q_{1,0}(x)=Q_{1,1}(x)=1$.

We call the polynomials $Q_{m, n}(x)$ the $q$-Okamoto polynomials. Some examples are as follows.

$$
\begin{aligned}
Q_{2,0}(x)= & x^{2}+\left(p^{2}+1\right) x+1 \\
Q_{3,0}(x)= & x^{6}+x^{5}\left(p^{6}+p^{4}+p^{2}+2+p^{-2}\right)+x^{4}\left(2 p^{8}+2 p^{6}+3 p^{4}+3 p^{2}+3+p^{-2}+p^{-4}\right) \\
& +x^{3}\left(p^{10}+2 p^{8}+3 p^{6}+4 p^{4}+4 p^{2}+3+2 p^{-2}+p^{-4}\right) \\
& +x^{2}\left(2 p^{8}+2 p^{6}+3 p^{4}+3 p^{2}+3+p^{-2}+p^{-4}\right)+x\left(p^{6}+p^{4}+p^{2}+2+p^{-2}\right)+1, \\
Q_{2,1}(x)= & x+1 \\
Q_{3,1}(x)= & x^{4}+x^{3}\left(p^{4}+p^{2}+1+p^{-2}\right)+x^{2}\left(2 p^{4}+p^{2}+2+p^{-2}\right)+x\left(p^{4}+p^{2}+1+p^{-2}\right)+1 .
\end{aligned}
$$

Similarly to the continuous case, $q$-Okamoto polynomials admit determinant formula of Jacobi-Trudi type. The proof of the Theorem 2.7 as well as the Jacobi-Trudi type formulas will be given in our next paper[9], where we will discuss the $\tau$ functions in a more general setting.

\section{Discussion}




\section{1 $\widetilde{W}\left(A_{2}^{(1)}\right) \times \widetilde{W}\left(A_{1}^{(1)}\right)$ symmetry and comparison with Sakai's Mul.6 system}

Along with the transformations $s_{i}$ and $\pi$ of $\widetilde{W}\left(A_{2}^{(1)}\right)$, we define the transformations $w_{0}, w_{1}$ and $r$ acting on $\mathbb{C}\left(a_{i}, f_{i}(i=0,1,2)\right)$ as follows:

$$
\begin{aligned}
& w_{0}\left(f_{0}\right)=\frac{a_{0} a_{1}\left(a_{2} a_{0}+a_{2} f_{0}+f_{2} f_{0}\right)}{f_{2}\left(a_{0} a_{1}+a_{0} f_{1}+f_{0} f_{1}\right)}, \quad w_{1}\left(f_{0}\right)=\frac{1+a_{0} f_{0}+a_{0} a_{1} f_{0} f_{1}}{a_{0} a_{1} f_{1}\left(1+a_{2} f_{2}+a_{2} a_{0} f_{2} f_{0}\right)}, \\
& w_{0}\left(f_{1}\right)=\frac{a_{1} a_{2}\left(a_{0} a_{1}+a_{0} f_{1}+f_{0} f_{1}\right)}{f_{0}\left(a_{1} a_{2}+a_{1} f_{2}+f_{1} f_{2}\right)}, \quad w_{1}\left(f_{1}\right)=\frac{1+a_{1} f_{1}+a_{1} a_{2} f_{1} f_{2}}{a_{1} a_{2} f_{2}\left(1+a_{0} f_{0}+a_{0} a_{1} f_{0} f_{1}\right)}, \\
& w_{0}\left(f_{2}\right)=\frac{a_{2} a_{0}\left(a_{1} a_{2}+a_{1} f_{2}+f_{1} f_{2}\right)}{f_{1}\left(a_{2} a_{0}+a_{2} f_{0}+f_{2} f_{0}\right)}, \quad w_{1}\left(f_{2}\right)=\frac{1+a_{2} f_{2}+a_{2} a_{0} f_{2} f_{0}}{a_{2} a_{0} f_{0}\left(1+a_{1} f_{1}+a_{1} a_{2} f_{1} f_{2}\right)}, \\
& r\left(f_{i}\right)=\frac{1}{f_{i}}, \quad w_{0}\left(a_{i}\right)=w_{1}\left(a_{i}\right)=r\left(a_{i}\right)=a_{i}, \quad(i=0,1,2) .
\end{aligned}
$$

Lemma 3.1 The transformations $w_{0}, w_{1}$ and $r$ generate the extended affine Weyl group $\widetilde{W}\left(A_{1}^{(1)}\right)$, namely we have

$$
w_{0}^{2}=w_{1}^{2}=r^{2}=1, \quad r w_{0}=w_{1} r .
$$

Moreover, this action of $\widetilde{W}\left(A_{1}^{(1)}\right)=\left\langle w_{0}, w_{1}, r\right\rangle$ commutes with that of $\widetilde{W}\left(A_{2}^{(1)}\right)=\left\langle s_{0}, s_{1}, s_{2}, \pi\right\rangle$.

Note that the discrete time evolution of the $q P_{I V}$ system is a translation of the $\widetilde{W}\left(A_{1}^{(1)}\right)$, that is,

$$
t=T_{4}=r w_{0} .
$$

Lemma 3.2 The representation of $\widetilde{W}\left(A_{2}^{(1)}\right) \times \widetilde{W}\left(A_{1}^{(1)}\right)$ is equivalent to Sakai's system Mul.6.

Proof. Let $x, y, z$ and $\tilde{a}_{0}, \tilde{a}_{1}, \tilde{a}_{2}, b_{1}, b_{0}=\tilde{a}_{0} \tilde{a}_{1} \tilde{a}_{2} / b_{1}$ be Sakai's homogeneous variables and the parameters 17. Then his representation of $\widetilde{W}\left(A_{2}^{(1)}\right) \times \widetilde{W}\left(A_{1}^{(1)}\right)$ is given as follows:

$$
\pi=\sigma^{4}, \quad s_{2}=\pi s_{1} \pi^{2}, \quad s_{0}=\pi^{2} s_{1} \pi, \quad r=\sigma^{3}, \quad w_{0}=r w_{1} r .
$$

Where $\sigma, s_{1}$ and $w_{1}$ is given by, I

$$
\begin{aligned}
& \sigma\left(\tilde{a}_{0}\right)=\tilde{a}_{1}, \quad \sigma\left(\tilde{a}_{1}\right)=\tilde{a}_{2}, \quad \sigma\left(\tilde{a}_{2}\right)=\tilde{a}_{0}, \quad \sigma\left(b_{0}\right)=b_{1}, \quad \sigma\left(b_{1}\right)=b_{0}, \\
& \sigma(x)=\tilde{a}_{2} x y(z-x), \quad \sigma(y)=-b_{1} y z(x+y-z), \quad \sigma(z)=\tilde{a}_{2} x(x-z)^{2}, \\
& s_{1}\left(\tilde{a}_{0}\right)=\tilde{a}_{0} \tilde{a}_{1}, \quad s_{1}\left(\tilde{a}_{1}\right)=\tilde{a}_{1}^{-1}, \quad s_{1}\left(\tilde{a}_{2}\right)=\tilde{a}_{2} \tilde{a}_{1}, \quad s_{1}\left(b_{i}\right)=b_{i}, \quad(i=0,1) \\
& s_{1}(x)=x, \quad s_{1}(y)=\tilde{a}_{1} y, \quad s_{1}(z)=\tilde{a}_{1} z, \\
& w_{1}\left(\tilde{a}_{i}\right)=\tilde{a}_{i}, \quad(i=0,1,2) \quad w_{1}\left(b_{0}\right)=b_{0} b_{1}^{2}, \quad w_{1}\left(b_{1}\right)=b_{1}^{-1}, \\
& w_{1}(x)=x\left(y+\tilde{a}_{2} x\right), \quad w_{1}(y)=-b_{1} y\left(y+\tilde{a}_{2} x\right), \quad w_{1}(z)=z\left(\tilde{a}_{2} x-b_{1} y\right) .
\end{aligned}
$$

Introduce variables $\tilde{f}_{i}(i=0,1,2)$ as

$$
\tilde{f}_{1}=-\frac{x}{z}, \quad \tilde{f}_{2}=\tilde{a}_{2} \frac{x-z}{y}, \quad \tilde{f}_{0}=\frac{b_{1} y z}{\tilde{a}_{2} x(x-z)} .
$$

Then we have

$$
\begin{aligned}
& \sigma\left(\tilde{f}_{0}\right)=\frac{\tilde{a}_{1}}{\tilde{f}_{1}}, \quad \sigma\left(\tilde{f}_{1}\right)=\frac{\tilde{a}_{2}}{\tilde{f}_{2}}, \quad \sigma\left(\tilde{f}_{2}\right)=\frac{\tilde{a}_{0}}{\tilde{f}_{0}}, \\
& s_{1}\left(\tilde{f}_{0}\right)=\tilde{f}_{0} \frac{\tilde{a}_{1}\left(1+\tilde{f}_{1}\right)}{\tilde{a}_{1}+\tilde{f}_{1}}, \quad s_{1}\left(\tilde{f}_{1}\right)=\frac{\tilde{f}_{1}}{\tilde{a}_{1}}, \quad s_{1}\left(\tilde{f}_{2}\right)=\frac{\tilde{a}_{1}+\tilde{f}_{2}}{1+\tilde{f}_{1}}, \\
& w_{1}\left(\tilde{f}_{0}\right)=\frac{1+\tilde{f}_{0}+\tilde{f}_{0} \tilde{f}_{1}}{\tilde{f}_{1}\left(1+\tilde{f}_{2}+\tilde{f}_{2} \tilde{f}_{0}\right)} \quad w_{1}\left(\tilde{f}_{1}\right)=\frac{1+\tilde{f}_{1}+\tilde{f}_{1} \tilde{f}_{2}}{\tilde{f}_{2}\left(1+\tilde{f}_{0}+\tilde{f}_{0} \tilde{f}_{1}\right)} \quad w_{1}\left(\tilde{f}_{2}\right)=\frac{1+\tilde{f}_{2}+\tilde{f}_{2} \tilde{f}_{0}}{\tilde{f}_{0}\left(1+\tilde{f}_{1}+\tilde{f}_{1} \tilde{f}_{2}\right)} .
\end{aligned}
$$

This representation is equivalent to our representation by the relation $\tilde{f}_{i}=a_{i} f_{i}, \quad \tilde{a}_{i}=a_{i}^{2}$.

\footnotetext{
${ }^{2}$ The generators $\sigma, s_{1}, w_{1}$ here correspond with $\sigma_{(123450)}, w_{1}$ and $w_{1}^{\prime}$ in [17]. An error in the formula of $\sigma_{(123450)}[17]$ is corrected.
} 


\subsection{Ultra-discretization of $q \mathbf{P}_{\mathrm{IV}}$}

In Section 1, we have mentioned that $q \mathrm{P}_{\mathrm{IV}}$ admit a continuous limit to the symmetric form of $\mathrm{P}_{\mathrm{IV}}$. There is another interesting limit, which is known as "ultra-discrete limit" [18]. We put

$$
f_{i}=\mathrm{e}^{F_{i} / \varepsilon}, \quad a_{i}=\mathrm{e}^{A_{i} / \varepsilon}, \quad i=0,1,2
$$

and take the limit $\varepsilon \rightarrow+0$. By using the formula,

$$
\lim _{\varepsilon \rightarrow+0} \varepsilon \log \left(\mathrm{e}^{\frac{A}{\varepsilon}}+\mathrm{e}^{\frac{B}{\varepsilon}}+\cdots\right)=\max (A, B, \cdots),
$$

$q \mathrm{P}_{\mathrm{IV}}$ (11) yields

$$
\begin{array}{ccc}
\bar{F}_{0}=t\left(F_{0}\right)=A_{0}+A_{1}+F_{1} & +\max \left(0, A_{2}+F_{2}, A_{0}+A_{2}+F_{0}+F_{2}\right) \\
& -\max \left(0, A_{0}+F_{0}, A_{1}+A_{0}+F_{1}+F_{0}\right), \\
\bar{F}_{1}=t\left(F_{1}\right)=A_{1}+A_{2}+F_{2} & +\max \left(0, A_{0}+F_{0}, A_{1}+A_{0}+F_{1}+F_{0}\right) \\
& -\max \left(0, A_{1}+F_{1}, A_{2}+A_{1}+F_{2}+F_{1}\right), \\
\bar{F}_{2}=t\left(F_{2}\right)=A_{2}+A_{0}+F_{0} & +\max \left(0, A_{1}+F_{1}, A_{2}+A_{1}+F_{2}+F_{1}\right) \\
& -\max \left(0, A_{2}+F_{2}, A_{0}+A_{2}+F_{0}+F_{2}\right), \\
\bar{A}_{i}=t\left(A_{i}\right)=A_{i}, &
\end{array}
$$

which we call the fourth ultra-discrete Painlevé equation $\left(\mathrm{uP}_{\mathrm{IV}}\right)$. Simultaneously, Bäcklund transformation (6) is ultra-discretized as

$$
\begin{aligned}
& s_{i}\left(F_{j}\right)=F_{j}+u_{i j}\left(\max \left(A_{i}, F_{i}\right)-\max \left(0, A_{i}+F_{i}\right)\right), \\
& s_{i}\left(A_{j}\right)=A_{j}-a_{i j} A_{i}, \quad \pi\left(X_{i}\right)=X_{i+1} \quad(i=0,1,2), \quad X=F, A
\end{aligned}
$$

where $A=\left(a_{i j}\right)_{i, j=0,1,2}$ and $U=\left(u_{i j}\right)_{i, j=0,1,2}$ are given by eq.(7). Then we can verify the following:

Proposition 3.3 The transformations $s_{0}, s_{1}, s_{2}$ and $\pi$ of the A-variables and the F-variables, defined by eq.(86) generate the extended affine Weyl group $\widetilde{W}=\left\langle s_{0}, s_{1}, s_{2}, \pi\right\rangle$ of type $A_{2}^{(1)}$. Furthermore, they commute with the time evolution $t$ of the fourth ultra-discrete Painleve equation $u P_{I V}$.

\section{A Difference Formulas and Plücker Relations}

In this appendix, we provide with data which are necessary for the proof of Proposition 2.4 .

We first note that it is possible to express $\phi_{N}^{\nu, m}$ in Section 2.2 as Casorati determinant with respect to $m$ as follows.

Lemma A.1 $\phi_{N}^{\nu, m}$ is rewritten as

$$
\begin{aligned}
\phi_{N}^{\nu, m} & =\left|\begin{array}{cccc}
G_{\nu}^{m} & G_{\nu-1}^{m} & \cdots & G_{\nu-N+1}^{m} \\
G_{\nu}^{m+1} & G_{\nu-1}^{m+1} & \cdots & G_{\nu-N+1}^{m+1} \\
\vdots & \vdots & \ddots & \vdots \\
G_{\nu}^{m+N-1} & G_{\nu-1}^{m+N-1} & \cdots & G_{\nu-N+1}^{m+N-1}
\end{array}\right| \\
\phi_{N}^{\nu, m} & =\prod_{k=1}^{N-1}\left(\frac{a_{0}^{2} q^{2(n-k)}}{a_{0}^{2} q^{2(n-k)}-1}\right)^{N-k}\left|\begin{array}{ccccc}
G_{\nu}^{m} & G_{\nu}^{m+1} & \cdots & G_{\nu}^{m+N-1} \\
G_{\nu}^{m-1} & G_{\nu}^{m} & \cdots & G_{\nu}^{m+N-2} \\
\vdots & \vdots & \ddots & \vdots \\
G_{\nu}^{m-N+1} & G_{\nu}^{m-N+2} & \cdots & G_{\nu}^{m}
\end{array}\right| .
\end{aligned}
$$

Proof of Lemma A.A. We use the contiguity relation (33), which is rewritten with the notation introduced in eq. (57) as

$$
G_{\nu}^{m+1}=G_{\nu+1}^{m}-q^{2 m} c^{2} G_{\nu}^{m} .
$$


For $k=2$ to $N$, subtracting $(j-1)$-st column multiplied by $q^{2 m} c^{2}$ from $j$-th column of eq.(58) for $j=N, \cdots k$, we obtain

$$
\begin{aligned}
\phi_{N}^{\nu, m} & =\left|\begin{array}{cccc}
G_{\nu}^{m} & G_{\nu+1}^{m}-q^{2 m} c^{2} G_{\nu}^{m} & \cdots & G_{\nu+N-1}^{m}-q^{2 m} c^{2} G_{\nu+N-2}^{m} \\
G_{\nu-1}^{m} & G_{\nu}^{m}-q^{2 m} c^{2} G_{\nu-1}^{m} & \cdots & G_{\nu-N-2}^{m}-q^{2 m} c^{2} G_{\nu+N-3}^{m} \\
\vdots & & \vdots & \ddots \\
G_{\nu-N+1}^{m} & G_{\nu-N+2}^{m}-q^{2 m} c^{2} G_{\nu-N+1}^{m} & \cdots & G_{\nu}^{m}-q^{2 m} c^{2} G_{\nu-1}^{m}
\end{array}\right| \\
& =\left|\begin{array}{cccc}
G_{\nu}^{m} & G_{\nu}^{m+1} & \cdots & G_{\nu+N-2}^{m+1} \\
G_{\nu-1}^{m} & G_{\nu-1}^{m+1} & \cdots & G_{\nu+N-3}^{m+1} \\
\vdots & \vdots & \ddots & \vdots \\
G_{\nu-N+1}^{m} & G_{\nu-N+1}^{m+1} & \cdots & G_{\nu-1}^{m+1}
\end{array}\right| \\
& =\left|\begin{array}{cccc}
G_{\nu}^{m} & G_{\nu}^{m+1} & \cdots & G_{\nu}^{m+N-1} \\
G_{\nu-1}^{m} & G_{\nu-1}^{m+1} & \cdots & G_{\nu-1}^{m+N-1} \\
\vdots & \vdots & \ddots & \vdots \\
G_{\nu-N+1}^{m} & G_{\nu-N+1}^{m+1} & \cdots & G_{\nu-N+1}^{m+N-1}
\end{array}\right|,
\end{aligned}
$$

which yields eq.(87) by taking the transposition. In order to derive eq.(88), we use the contiguity relation,

$$
G_{\nu-1}^{m}+\frac{G_{\nu}^{m}}{a_{0}^{2} q^{2(\nu-1)}-1}=\frac{a_{0}^{2} q^{2(\nu-1)}}{a_{0}^{2} q^{2(\nu-1)}-1} G_{\nu}^{m-1},
$$

which is obtained from eqs. (32) and (33). For $k=2$ to $N$, subtracting $(i-1)$-st row multiplied by $\frac{1}{a_{0}^{2} q^{2(\nu-i-1)}-1}$ from $i$-th row of eq.(90) for $i=N, \cdots k$, we obtain

$$
\begin{aligned}
& \phi_{N}^{\nu, m}=\left|\begin{array}{cccc}
G_{\nu}^{m} & G_{\nu}^{m+1} & \cdots & G_{\nu}^{m+N-1} \\
\vdots & \vdots & \ddots & \vdots \\
G_{\nu-N+2}^{m} & G_{\nu-N+2}^{m+1} & \cdots & G_{\nu-N+2}^{m+N-1} \\
G_{\nu-N+1}^{m}-\frac{G_{\nu-N+2}^{m}}{a_{0}^{2} q^{2(\nu-N+1)}-1} & G_{\nu-N+1}^{m+1}-\frac{G_{\nu+N+2}^{m+1}}{a_{0}^{2} q^{2(\nu-N+1)}-1} & \cdots & G_{\nu-N+1}^{m+N-1}-\frac{G_{\nu+N+2}^{m+N-1}}{a_{0}^{2} q^{(2-N+1)}-1}
\end{array}\right| \\
& =\frac{a_{0}^{2} q^{2(\nu-N+1)}}{a_{0}^{2} q^{2(\nu-N+1)}-1}\left|\begin{array}{cccc}
G_{\nu}^{m} & G_{\nu}^{m+1} & \cdots & G_{\nu}^{m+N-1} \\
\vdots & \vdots & \ddots & \vdots \\
G_{\nu-N+2}^{m} & G_{\nu-N+2}^{m+1} & \cdots & G_{\nu-N-1}^{m+N+2} \\
G_{\nu-N+2}^{m-1} & G_{\nu-N+2}^{m} & \cdots & G_{\nu-N+2}^{m+N-2}
\end{array}\right| \\
& =\cdots \\
& =\prod_{i=1}^{N-1} \frac{a_{0}^{2} q^{2(\nu-i)}}{a_{0}^{2} q^{2(\nu-i)}-1}\left|\begin{array}{cccc}
G_{\nu}^{m} & G_{\nu}^{m+1} & \cdots & G_{\nu}^{m+N-1} \\
G_{\nu}^{m-1} & G_{\nu}^{m} & \cdots & G_{\nu}^{m+N-2} \\
\vdots & \vdots & \ddots & \vdots \\
G_{\nu-N+2}^{m} & G_{\nu-N+2}^{m+1} & \cdots & G_{\nu-N-1}^{m+N+2} \\
G_{\nu-N+2}^{m-1} & G_{\nu-N+2}^{m} & \cdots & G_{\nu-N+2}^{m+N+N}
\end{array}\right| \\
& =\cdots \\
& =\prod_{i=1}^{N-1}\left(\frac{a_{0}^{2} q^{2(\nu-i)}}{a_{0}^{2} q^{2(\nu-i)}-1}\right)^{N-i}\left|\begin{array}{cccc}
G_{\nu}^{m} & G_{\nu}^{m+1} & \cdots & G_{\nu}^{m+N-1} \\
G_{\nu}^{m-1} & G_{\nu}^{m} & \cdots & G_{\nu}^{m+N-2} \\
\vdots & \vdots & \ddots & \vdots \\
G_{\nu}^{m-N+2} & G_{\nu}^{m-N+3} & \cdots & G_{\nu}^{m-1} \\
G_{\nu}^{m-N+1} & G_{\nu}^{m-N} & \cdots & G_{\nu}^{m}
\end{array}\right|
\end{aligned}
$$

which is eq.(88). 
In view of this lemma, we introduce the following notations.

$$
\begin{aligned}
& \phi_{N}^{\nu, m}=\left|\begin{array}{cccc}
G_{\nu}^{m} & G_{\nu+1}^{m} & \cdots & G_{\nu+N-1}^{m} \\
G_{\nu-1}^{m} & G_{\nu}^{m} & \cdots & G_{\nu-N-2}^{m} \\
\vdots & \vdots & \ddots & \vdots \\
G_{\nu-N+1}^{m} & G_{\nu-N+2}^{m} & \cdots & G_{\nu}^{m}
\end{array}\right|=\left|\mathbf{0}^{m}, \mathbf{1}^{m}, \cdots, \mathbf{N}-\mathbf{1}^{m}\right|, \\
& \phi_{N}^{\nu, m}=\left|\begin{array}{cccc}
G_{\nu}^{m} & G_{\nu-1}^{m} & \cdots & G_{\nu-N+1}^{m} \\
G_{\nu}^{m+1} & G_{\nu-1}^{m+1} & \cdots & G_{\nu-N+1}^{m+1} \\
\vdots & \vdots & \ddots & \vdots \\
G_{\nu}^{m+N-1} & G_{\nu-1}^{m+N-1} & \cdots & G_{\nu-N+1}^{m+N-1}
\end{array}\right|=\left|\underline{\mathbf{0}}^{m-1}, \underline{-1}^{m-1}, \cdots, \underline{-\mathbf{N}+\mathbf{1}^{m-1}}\right|,
\end{aligned}
$$

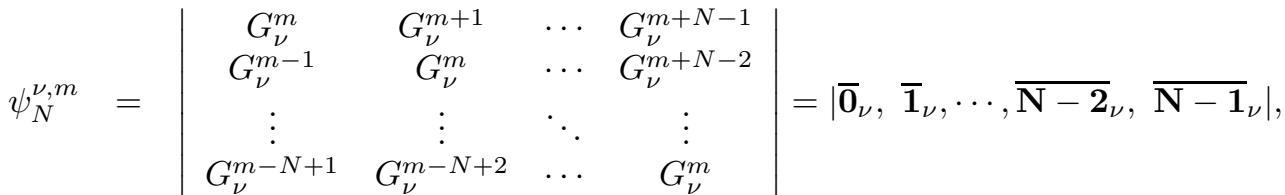

where $\mathbf{k}^{m}, \underline{\mathbf{k}}^{m}$ and $\overline{\mathbf{k}}_{\nu}$ are column vectors given by

$$
\mathbf{k}^{m}=\left(\begin{array}{c}
G_{\nu+k}^{m} \\
G_{\nu+k-1}^{m} \\
\vdots \\
G_{\nu+k-N+1}^{m}
\end{array}\right), \quad \underline{\mathbf{k}}^{m}=\left(\begin{array}{c}
G_{\nu+k}^{m} \\
G_{\nu+k}^{m+1} \\
\vdots \\
G_{\nu+k}^{m+N-1}
\end{array}\right), \quad \overline{\mathbf{k}}_{\nu}=\left(\begin{array}{c}
G_{\nu}^{m+k} \\
G_{\nu}^{m+k-1} \\
\vdots \\
G_{\nu}^{m+k-N+1}
\end{array}\right)
$$

respectively. We also use auxiliary column vectors,

$$
\mathbf{k}^{m \prime}=\left(\begin{array}{c}
G_{\nu+k}^{m} \\
q^{2} G_{\nu+k-1}^{m} \\
\vdots \\
q^{2(N-1)} G_{\nu+k-N+1}^{m}
\end{array}\right), \quad \underline{\mathbf{k}}^{m \dagger}=\left(\begin{array}{c}
G_{\nu+k}^{m} \\
q^{-2} G_{\nu+k}^{m+1} \\
\vdots \\
q^{-2(N-1)} G_{\nu+k}^{m+N-1}
\end{array}\right), \quad \overline{\mathbf{k}}_{\nu}^{\prime}=\left(\begin{array}{c}
G_{\nu}^{m+k} \\
q^{2} G_{\nu}^{m+k-1} \\
\vdots \\
q^{2(N-1)} G_{\nu}^{m+k-N+1}
\end{array}\right) .
$$

Now we give difference formulas.

\section{Difference Formula I}

$$
\begin{aligned}
& \left|\mathbf{0}^{m+1 \prime}, \mathbf{0}^{m}, \mathbf{1}^{m}, \cdots, \mathbf{N}-\mathbf{3}^{m}, \mathbf{N}-\mathbf{2}^{m}\right|=a_{0}^{-2(N-1)} c^{-2(N-1)} q^{-2(N-1)(m+\nu-1)} \phi_{N}^{\nu, m+1}, \\
& \left|\mathbf{1}^{m+1 \prime}, \mathbf{0}^{m}, \mathbf{1}^{m}, \cdots, \mathbf{N}-\mathbf{3}^{m}, \mathbf{N}-\mathbf{2}^{m}\right|=a_{0}^{-2(N-1)} c^{-2(N-1)} q^{-2(N-1)(m+\nu-1)} \phi_{N}^{\nu, m+1} .
\end{aligned}
$$

\section{Difference Formula II}

$$
\begin{aligned}
& \left|\mathbf{0}^{m}, \mathbf{0}^{m+1}, \mathbf{1}^{m+1}, \cdots, \mathbf{N}-\mathbf{3}^{m+1}, \mathbf{N}-\mathbf{2}^{m+1}\right|=\phi_{N}^{\nu, m}, \\
& \left|\mathbf{1}^{m}, \mathbf{0}^{m+1}, \mathbf{1}^{m+1}, \cdots, \mathbf{N}-\mathbf{3}^{m+1}, \mathbf{N}-\mathbf{2}^{m+1}\right|=c^{2} q^{2 m} \phi_{N}^{\nu, m} .
\end{aligned}
$$

\section{Difference formula III}

$$
\begin{aligned}
& \left|\underline{\mathbf{1}}^{m-1}, \underline{\mathbf{- 2}}^{m-1}, \cdots, \underline{\underline{-N}+\mathbf{1}^{m-1}}, \underline{-\mathbf{N}+\mathbf{1}^{m \dagger}}\right|=a_{0}^{-2(N-1)} c^{-2(N-1)} q^{-2(N-1)(\nu+m-1)} \phi_{N}^{\nu, m}, \\
& \left|\underline{\mathbf{- 1}}^{m-1}, \underline{\mathbf{- 2}}^{m-1}, \cdots, \underline{\mathbf{- N}+\mathbf{1}^{m-1}}, \underline{-\mathbf{N}+\mathbf{2}^{m \dagger}}\right|=a_{0}^{-2(N-1)} c^{-2(N-1)} q^{-2(N-1)(\nu+m-1)} \phi_{N}^{\nu, m} .
\end{aligned}
$$

Difference Formula IV

$$
\begin{aligned}
& \left|\overline{\mathbf{0}}_{\nu+1}^{\prime}, \overline{\mathbf{0}}_{\nu}, \cdots, \overline{\mathbf{N}-\mathbf{2}}_{\nu}\right|=c^{-2(N-1)}\left(a_{0}^{2} q^{2 \nu}-1\right)^{-(N-1)} q^{-2(m-1)(N-1)} \psi_{N}^{\nu+1, m}, \\
& \left|\overline{\mathbf{1}}_{\nu+1}^{\prime}, \overline{\mathbf{0}}_{\nu}, \cdots, \overline{\mathbf{N}}-\mathbf{2}_{\nu}\right|=c^{-2(N-1)}\left(a_{0}^{2} q^{2 \nu}-1\right)^{-(N-1)} q^{-2(m-1)(N-1)} \psi_{N}^{\nu+1, m} .
\end{aligned}
$$

\section{Difference Formula V}

$$
\begin{aligned}
\mid \overline{\mathbf{0}}_{\nu+1}, \overline{\mathbf{0}}_{\nu}, \cdots, \overline{\mathbf{N}-\mathbf{2}} & =\left(1-a_{0}^{2} q^{2 \nu}\right)^{-(N-1)} \psi_{N}^{\nu+1, m} \\
\mid \overline{\mathbf{1}}_{\nu+1}, & \overline{\mathbf{0}}_{\nu}, \cdots, \overline{\mathbf{N}-\mathbf{2}}_{\nu} \mid=\left(1-a_{0}^{2} q^{2 \nu}\right)^{-(N-1)} a_{0}^{2} q^{2 \nu} \psi_{N}^{\nu+1, m}
\end{aligned}
$$


Table 1: Data for the proof of difference formulas

\begin{tabular}{|c||c|c|}
\hline Difference Formula & Contiguity Relation & Determinant \\
\hline Difference Formula I (97) & $G_{\nu+1}^{m+1}=G_{\nu}^{m+1}+a_{0}^{2} q^{2(\nu+m)} c^{2} G_{\nu}^{m}$ & eq.(92) \\
\hline Difference Formula II(98) & $G_{\nu}^{m+1}=G_{\nu+1}^{m}-q^{2 m} c^{2} G_{\nu}^{m}$ & eq.(92) \\
\hline Difference Formula III(99) & $G_{\nu}^{m}-G_{\nu-1}^{m}=a_{0}^{2} c^{2} q^{2(\nu+m-2)} G_{\nu-1}^{m-1}$ & eq.(93) \\
\hline Difference Formula IV(100) & $G_{\nu+1}^{m+1}-G_{\nu+1}^{m}=c^{2} q^{2 m}\left(1-a_{0} q^{2 \nu}\right) G_{\nu}^{m}$ & eq.(94) \\
\hline Difference Formula $\mathrm{V}(101)$ & $G_{\nu+1}^{m+1}-a_{0} q^{2 \nu} G_{\nu+1}^{m}=\left(1-a_{0} q^{2 \nu}\right) G_{\nu}^{m+1}$ & eq.(94) \\
\hline
\end{tabular}

Difference formula I has been proved in Lemma 2.5. Other formulas are proved in a similar manner. Table 1 shows the the contiguity relation and determinant to be used for the derivation of each difference formula.

Next we give the list of Plücker relations which are necessary for the proof of Proposition 2.4.

\section{Plücker Relation I}

$$
\begin{aligned}
0 & =\left|\varphi_{1}, \mathbf{1}^{m+1 \prime}, \mathbf{1}^{m}, \cdots, \mathbf{N}-\mathbf{1}^{m}\right| \times\left|\mathbf{0}^{m}, \mathbf{1}^{m}, \cdots, \mathbf{N}-\mathbf{1}^{m}, \mathbf{N}^{m}\right| \\
& -\left|\varphi_{1}, \mathbf{0}^{m}, \mathbf{1}^{m}, \cdots, \mathbf{N}-\mathbf{1}^{m}\right| \times\left|\mathbf{1}^{m+1 \prime}, \mathbf{1}^{m}, \cdots, \mathbf{N}-\mathbf{1}^{m}, \mathbf{N}^{m}\right| \\
& +\left|\mathbf{1}^{m+1 \prime}, \mathbf{0}^{m}, \mathbf{1}^{m}, \cdots, \mathbf{N}-\mathbf{1}^{m}\right| \times\left|\varphi_{1}, \mathbf{1}^{m}, \cdots, \mathbf{N}-\mathbf{1}^{m}, \mathbf{N}^{m}\right|
\end{aligned}
$$

\section{Plücker Relation II}

$$
\begin{aligned}
0 & =\left|\varphi_{2}, \mathbf{1}^{m+1 \prime}, \mathbf{1}^{m}, \cdots, \mathbf{N}-\mathbf{1}^{m}\right| \times\left|\mathbf{0}^{m}, \mathbf{1}^{m}, \cdots, \mathbf{N}-\mathbf{1}^{m}, \mathbf{N}^{m}\right| \\
& -\left|\varphi_{2}, \mathbf{0}^{m}, \mathbf{1}^{m}, \cdots, \mathbf{N}-\mathbf{1}^{m}\right| \times\left|\mathbf{1}^{m+1}, \mathbf{1}^{m}, \cdots, \mathbf{N}-\mathbf{1}^{m}, \mathbf{N}^{m}\right| \\
& +\left|\mathbf{1}^{m+1 \prime}, \mathbf{0}^{m}, \mathbf{1}^{m}, \cdots, \mathbf{N}-\mathbf{1}^{m}\right| \times\left|\varphi_{2}, \mathbf{1}^{m}, \cdots, \mathbf{N}-\mathbf{1}^{m}, \mathbf{N}^{m}\right|
\end{aligned}
$$

\section{Plücker Relation III}

$$
\begin{aligned}
0 & =\left|\varphi_{2}, \mathbf{1}^{m}, \mathbf{1}^{m+1}, \cdots, \mathbf{N}-\mathbf{1}^{m+1}\right| \times\left|\mathbf{0}^{m+1}, \mathbf{1}^{m+1}, \cdots, \mathbf{N}-\mathbf{1}^{m+1}, \mathbf{N}^{m+1}\right| \\
& -\left|\varphi_{2}, \mathbf{0}^{m+1}, \mathbf{1}^{m+1}, \cdots, \mathbf{N}-\mathbf{1}^{m+1}\right| \times\left|\mathbf{1}^{m}, \mathbf{1}^{m+1}, \cdots, \mathbf{N}-\mathbf{1}^{m+1}, \mathbf{N}^{m+1}\right| \\
& +\left|\mathbf{1}^{m}, \mathbf{0}^{m+1}, \mathbf{1}^{m+1}, \cdots, \mathbf{N}-\mathbf{1}^{m+1}\right| \times\left|\varphi_{2}, \mathbf{1}^{m+1}, \cdots, \mathbf{N}-\mathbf{1}^{m+1}, \mathbf{N}^{m+1}\right|
\end{aligned}
$$

\section{Plücker Relation IV}

$$
\begin{aligned}
0 & =\left|\varphi_{2}, \varphi_{1}, \mathbf{1}^{m+1}, \cdots, \mathbf{N}-\mathbf{1}^{m+1}\right| \times\left|\mathbf{1}^{m}, \mathbf{1}^{m+1}, \cdots, \mathbf{N}-\mathbf{1}^{m+1}, \mathbf{N}^{m+1}\right| \\
& -\left|\varphi_{2}, \mathbf{1}^{m}, \mathbf{1}^{m+1}, \cdots, \mathbf{N}-\mathbf{1}^{m+1}\right| \times\left|\varphi_{1}, \mathbf{1}^{m+1}, \cdots, \mathbf{N}-\mathbf{1}^{m+1}, \mathbf{N}^{m+1}\right| \\
& +\left|\varphi_{1}, \mathbf{1}^{m}, \mathbf{1}^{m+1}, \cdots, \mathbf{N}-\mathbf{1}^{m+1}\right| \times\left|\varphi_{2}, \mathbf{1}^{m+1}, \cdots, \mathbf{N}-\mathbf{1}^{m+1}, \mathbf{N}^{m+1}\right|
\end{aligned}
$$

\section{Plücker Relation V}

$$
\begin{aligned}
0 & =\left|\varphi_{2}, \varphi_{1}, \mathbf{0}^{m}, \cdots, \mathbf{N}-\mathbf{2}^{m}\right| \times\left|\mathbf{0}^{m+1 \prime}, \mathbf{0}^{m}, \cdots, \mathbf{N}-\mathbf{2}^{m}, \mathbf{N}-\mathbf{1}^{m}\right| \\
& -\left|\varphi_{2}, \mathbf{0}^{m+1 \prime}, \mathbf{0}^{m}, \cdots, \mathbf{N}-\mathbf{2}^{m}\right| \times\left|\varphi_{1}, \mathbf{0}^{m}, \cdots, \mathbf{N}-\mathbf{2}^{m}, \mathbf{N}-\mathbf{1}^{m}\right| \\
& +\left|\varphi_{1}, \mathbf{0}^{m+1 \prime}, \mathbf{0}^{m}, \cdots, \mathbf{N}-\mathbf{2}^{m}\right| \times\left|\varphi_{2}, \mathbf{0}^{m}, \cdots, \mathbf{N}-\mathbf{2}^{m}, \mathbf{N}-\mathbf{1}^{m}\right|
\end{aligned}
$$

\section{Plücker Relation VI}

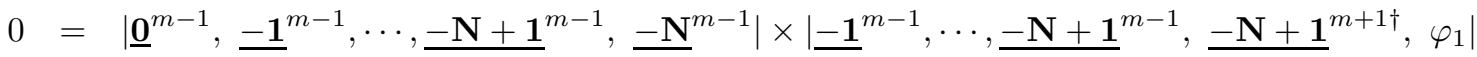

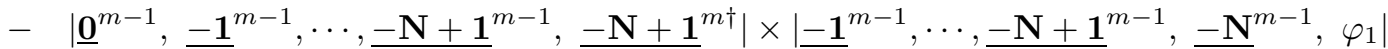

$$
\begin{aligned}
& +\left|\underline{\mathbf{0}}^{m-1}, \underline{\mathbf{- 1}}^{m-1}, \cdots, \underline{\mathbf{- N}+\mathbf{1}^{m-1}}, \varphi_{1}\right| \times\left|\underline{\mathbf{- 1}}^{m-1}, \cdots, \underline{-\mathbf{N}+\mathbf{1}^{m-1}}, \underline{-\mathbf{N}}^{m-1}, \underline{\mathbf{- N}+\mathbf{1}^{m \dagger}}\right| \text {. }
\end{aligned}
$$




\section{Plücker Relation VII}

$$
\begin{aligned}
0 & =\left|\varphi_{1}, \overline{\mathbf{1}}_{\nu+1}^{\prime}, \overline{\mathbf{1}}_{\nu}, \cdots, \overline{\mathbf{N}-\mathbf{1}_{\nu}}\right| \times\left|\overline{\mathbf{0}}_{\nu}, \overline{\mathbf{1}}_{\nu}, \cdots, \overline{\mathbf{N}-\mathbf{1}_{\nu}}, \overline{\mathbf{N}}_{\nu}\right| \\
& -\left|\varphi_{1}, \overline{\mathbf{0}}_{\nu}, \overline{\mathbf{1}}_{\nu}, \cdots,{\overline{\mathbf{N}-\mathbf{1}_{\nu}}}\right| \times\left|\overline{\mathbf{1}}_{\nu+1}^{\prime}, \overline{\mathbf{1}}_{\nu}, \cdots,{\overline{\mathbf{N}-\mathbf{1}_{\nu}}}_{\nu}, \overline{\mathbf{N}}_{\nu}\right| \\
& +\left|\overline{\mathbf{1}}_{\nu+1}^{\prime}, \overline{\mathbf{0}}_{\nu}, \overline{\mathbf{1}}_{\nu}, \cdots,{\overline{\mathbf{N}-\mathbf{1}_{\nu}}}_{\nu}\right| \times\left|\varphi_{1}, \overline{\mathbf{1}}_{\nu}, \cdots,{\overline{\mathbf{N}-\mathbf{1}_{\nu}}}_{\nu}, \overline{\mathbf{N}}_{\nu}\right|
\end{aligned}
$$

\section{Plücker Relation VIII}

$$
\begin{aligned}
& 0=\left|\varphi_{2}, \varphi_{1}, \overline{\mathbf{1}}_{\nu}, \cdots,{\overline{\mathbf{N}-\mathbf{1}_{\nu}}}_{\nu}\right| \times\left|\overline{\mathbf{0}}_{\nu+1}, \overline{\mathbf{1}}_{\nu}, \cdots, \overline{\mathbf{N}-\mathbf{1}_{\nu}}, \overline{\mathbf{N}}_{\nu}\right| \\
& \text { - }\left|\varphi_{2}, \overline{\mathbf{0}}_{\nu+1}, \overline{\mathbf{1}}_{\nu}, \cdots, \overline{\mathbf{N}-\mathbf{1}_{\nu}}\right| \times\left|\varphi_{1}, \overline{\mathbf{1}}_{\nu}, \cdots, \overline{\mathbf{N}-\mathbf{1}_{\nu}}, \overline{\mathbf{N}}_{\nu}\right|
\end{aligned}
$$

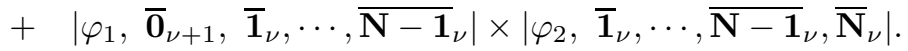

Table 2: Data for the proof of bilinear equations

\begin{tabular}{|c||l|l|}
\hline Bilinear Equation & Plücker Relation & Difference Formula \\
\hline eq.(42) & Plücker Relation I (102) & Difference Formula I (97) \\
\hline \multirow{2}{*}{ eqs.(43) and (44) } & Plücker Relation II (103) & Difference Formula I (97) \\
& Plücker Relation III (104) & Difference Formula II (98) \\
\hline eq.(45) & Plücker Relation IV(105) & Difference Formula II (98) \\
\hline eq.(46) & Plücker Relation V(106) & Difference Formula I (97) \\
\hline eq.(47) & Plücker Relation VI(107) & Difference Formula III (99) \\
\hline eq.(48) & Plücker Relation VII(108) & Difference Formula IV (100) \\
\hline eq.(49) & Plücker Relation VIII(109) & Difference Formula V (101) \\
\hline
\end{tabular}

Here, $\varphi_{i}(i=1,2)$ are any column vectors which we specialize as

$$
\varphi_{1}=\left(\begin{array}{c}
1 \\
0 \\
\vdots \\
0
\end{array}\right), \quad \varphi_{2}=\left(\begin{array}{c}
0 \\
\vdots \\
0 \\
1
\end{array}\right)
$$

in the proof of bilinear equations.

In Section 2.2.2, we have shown how eq.(42) can be derived from the Plücker relation I and difference formula I. The other bilinear equations in Proposition 2.4 are obtained similarly by using the data described in Table 2.

\section{References}

[1] V.E. Adler: Nonlinear chains and Painlevé equations, Physica D 73(1994), 335-351.

[2] G. Gasper and M. Rahman: Basic Hypergeometric Series, Encyclopedia of Mathematics and its Applications, 35. Cambridge University Press, Cambridge (1990).

[3] B. Grammaticos, F. Nijhoff and A. Ramani: Discrete Painlevé equations, in "The Painlevé Property - One Century Later" (Ed. R. Conte), CRM Series in Mathematical Physics, Springer-Verlag, New York, 1999, $413-516$.

[4] B. Grammaticos, A. Ramani and V. G. Papageorgiou: Do integrable mappings have the Painlevé property?, Phys. Rev. Lett.67(1991), 1825-1828. 
[5] B. Grammaticos and A. Ramani: Discrete Painlevé equations: coalescences, limits and degeneracies, PhyicaA228(1996), 160-171.

[6] M. Jimbo, T. Miwa and K. Ueno: Monodromy preserving deformation of linear ordinary differential equations with rational coefficients, Physica 2D(1981), 306-352.

[7] M. Jimbo and T. Miwa : Monodromy preserving deformation of linear ordinary differential equations with rational coefficients II, Physica 2D(1981), 407-448.

[8] M. Jimbo and T. Miwa : Monodromy preserving deformation of linear ordinary differential equations with rational coefficients III, Physica 3D(1981), 26-46.

[9] K. Kajiwara, M. Noumi and Y. Yamada: in preparation.

[10] K. Kajiwara and Y. Ohta: Determinant structure of the rational solutions for the Painlevé IV equation, J. Phys. A 31(1998), 2431-2446.

[11] R. Koekoek and R. F. Swarttouw: The Askey-scheme of hypergeometric orthogonal polynomials and its $q$-analogue, Delft University of Technology, Department of Technical Mathematics and Informatics Report no. 98-17, 1998.

[12] M. Noumi and Y. Yamada: Affine Weyl groups, discrete dynamical systems and Painlevé equations, Commun. Math. Phys. 199(1998), 281-195.

[13] M. Noumi and Y. Yamada: Higher order Painlevé equations of type $A_{l}^{(1)}$, Funkcial. Ekvac. 41(1998), 483503.

[14] M. Noumi and Y. Yamada: Symmetries in the fourth Painlevé equation and Okamoto polynomials, Nagoya Math. J. 153(1999), 53-86.

[15] K. Okamoto: Studies on the Painlevé equations. III. Second and Fourth Painlevé equations, $\mathrm{P}_{\mathrm{II}}$ and $\mathrm{P}_{\mathrm{IV}}$., Math. Ann. 275(1986), 221-255.

[16] A. Ramani, B. Grammaticos and J. Hietarinta: Discrete versions of the Painlevé equations, Phys. Rev. Lett. 67(1991), 1829-1832.

[17] H. Sakai: Rational surfaces associated with affine root systems and geometry of the Painlevé equations, preprint(Kyoto-Math 99-10, Kyoto Univ., 1999).

[18] T. Tokihiro, D. Takahashi, J. Matsukidaira and J. Satsuma: From soliton equations to integrable cellular automata through a limiting procedure, Phys. Rev. Lett. 76(1996), 3247-3250.

[19] A.P. Veselov and A.B. Shabat: A dressing chain and the spectral theory of the Shrödinger operator, Funct. Anal. Appl. 27(1993), 1-21. 